\title{
FCNC decays of SM fermions into a dark photon
}

\author{
Emidio Gabrielli $^{a, b}$, Barbara Mele $^{c}$, Martti Raidal $^{b, d}$, and Elena Venturini ${ }^{e}$ \\ (a) Dipart. di Fisica Teorica, Università di Trieste, Strada Costiera 11, I-34151 Trieste, Italy \\ and INFN, Sezione di Trieste, Via Valerio 2, I-34127 Trieste, Italy \\ (b) NICPB, Rävala 10, 10143 Tallinn, Estonia \\ (c) INFN, Sezione di Roma, c/o Dipart. di Fisica, Università di Roma "La Sapienza", \\ Piazzale Aldo Moro 2, I-00185 Rome, Italy \\ (d) Institute of Physics, University of Tartu, Ravila 14c, 50411 Tartu, Estonia \\ (e) SISSA/ISAS and INFN, Via Bonomea 265, I-34136 Trieste, Italy
}

\begin{abstract}
We analyze a new class of FCNC processes, the $f \rightarrow f^{\prime} \bar{\gamma}$ decays of a fermion $f$ into a lighter (same-charge) fermion $f^{\prime}$ plus a massless neutral vector boson, a dark photon $\bar{\gamma}$. A massless dark photon does not interact at tree level with observable fields, and the $f \rightarrow f^{\prime} \bar{\gamma}$ decay presents a characteristic signature where the final fermion $f^{\prime}$ is balanced by a massless invisible system. Models recently proposed to explain the exponential spread in the standard-model Yukawa couplings can indeed foresee an extra unbroken dark $U(1)$ gauge group, and the possibility to couple on-shell dark photons to standard-model fermions via one-loop magnetic-dipole kind of FCNC interactions. The latter are suppressed by the characteristic scale related to the mass of heavy messengers, connecting the standard model particles to the dark sector. We compute the corresponding decay rates for the top, bottom, and charm decays $(t \rightarrow c \bar{\gamma}, u \bar{\gamma}, b \rightarrow s \bar{\gamma}, d \bar{\gamma}$, and $c \rightarrow u \bar{\gamma})$, and for the charged-lepton decays $(\tau \rightarrow \mu \bar{\gamma}, e \bar{\gamma}$, and $\mu \rightarrow e \bar{\gamma})$ in terms of model parameters. We find that large branching ratios for both quark and lepton decays are allowed in case the messenger masses are in the discovery range of the LHC. Implications of these new decay channels at present and future collider experiments are briefly discussed.
\end{abstract}




\section{Introduction}

One of the most intriguing aspects of the standard model (SM) is the nontrivial structure of the flavor sector, which is encoded in the corresponding structure of the Higgs-boson Yukawa couplings. The latter seem not to be originating from any global or gauge symmetry, and resemble effective couplings rather than fundamental ones, their eigenvalues spanning over almost 6 orders of magnitude for charged fermions, and much more in case neutrinos are Dirac particles. The Cabibbo-Kobayashi-Maskawa (CKM) mixing matrix in the quark sector of weak charged currents (and the analogous one in the leptonic sector) adds further mystery to the origin and structure of flavor.

The recent discovery of the Higgs boson [1] has strengthened our confidence in the Higgs mechanism [2], and in the existence of its Yukawa couplings to fermions, necessary for the fermion mass generation mechanism in the SM framework. All the observed Higgs properties seem to be in good agreement with the SM predictions [3], although there is still large room for potential new physics (NP) contributions. In this respect, the present experimental situation does not help, yet, to clarify whether the Yukawa couplings are fundamental or low-energy effective couplings, leaving space for new conjectures about the true origin of flavor.

In case the Yukawa couplings are not fundamental, an interesting possibility is to conjecture that the chiral symmetry breaking $(\mathrm{ChSB})$ and flavor structure originate from a dark sector and is communicated to the SM by some kind of messenger fields [4, 5]. The latter are by definition fields that couple both to the SM and dark-sector fields at tree level. Then, due to the messenger interactions, the Yukawa couplings can be generated at one loop as effective low-energy couplings.

In this paper, we focus on the recent proposal in [4], aiming at solving the flavor hierarchy problem by explaining the exponential spread in the Yukawa couplings at low energy. For each SM fermion, this model predicts the existence of a massive fermion partner in the dark sector, singlet under the SM gauge group (dubbed dark fermion in the following), and a set of scalar messenger fields carrying the same SM quantum numbers of squarks and slepton in supersymmetric models. The Yukawa couplings $Y_{f}$ (where $f$ is a flavor index) are required to be vanishing at tree level by imposing a discrete Higgs $(H)$ parity, $H \rightarrow-H$. Then, via the spontaneous breaking of this symmetry, Yukawa couplings can be generated at one loop. In particular, they can be induced by universal trilinear interactions that mix SM fields, dark fermions and messenger fields. Due to chirality, the resulting Yukawa couplings turn out to be proportional to dark fermion masses $M_{F_{f}}$,

$$
Y_{f} \sim \frac{M_{F_{f}}}{\Lambda_{\text {eff }}},
$$

where $\Lambda_{\text {eff }}$ is an (almost) flavor-universal effective scale. As a consequence, the observed SM Yukawa hierarchy just reflects the structure of the dark fermion spectrum 1 ,

However, this conjecture alone is not sufficient to naturally solve the SM flavor hierarchy problem. A new dynamical mechanism is needed to explain the required pattern of dark-fermion

\footnotetext{
${ }^{1} \mathrm{~A}$ similar scenario with radiatively generated Yukawa couplings, and a $Y_{f}$ pattern as in Eq. (1) has been proposed in [5], although the latter does not include a discussion of the dynamics responsible for the dark-fermion mass spectrum needed to give rise to the SM Yukawa hierarchy.
} 
masses. In [4], a nonperturbative mechanism has been proposed to generate exponentially spread dark-fermion masses. It requires the existence of an exact $U(1)_{F}$ gauge symmetry in the dark sector, and dark fermions $F_{f}$ charged under $U(1)_{F}$ with $\bar{e}_{f}$ quantum charges [in units of the fundamental $U(1)_{F}$ charge $\left.\bar{e}\right]$. In particular, this mechanism, based on a Nambu-JonaLasinio approach [6], predicts exponentially spread masses $M_{F_{f}}$ for dark fermions according to the law [4]

$$
M_{F_{f}}=\Lambda \exp \left(-\frac{\gamma}{\bar{\alpha} \bar{e}_{f}^{2}}\right),
$$

where $\bar{\alpha}=\bar{e}^{2} /(4 \pi)$ is the $U(1)_{F}$ fine structure constant, and $\gamma$ is connected to an anomalous dimension. The $\Lambda$ scale is associated to the Lee-Wick term for the $U(1)_{F}$ gauge sector [7, 8], which is responsible for triggering spontaneous $\mathrm{ChSB}$, and generating Dirac fermion masses [4].

The nonperturbative origin of the spectrum in Eq. (2) as a function of $\bar{\alpha}$, is shown by the $1 / \bar{\alpha}$ dependence in the exponent. Then, by assuming order- $\mathcal{O}(1)$ nonuniversality among the $U(1)_{F}$ dark-fermion charges $\bar{e}_{f}$, a wide exponential spread among fermion masses can be easily generated. Then Eq. (2), along with Eq.(1), can provide the theoretical basis for a natural solution to the SM flavor hierarchy problem.

A peculiar aspect of this model is the existence of a dark photon associated to the unbroken $U(1)_{F}$ gauge field, which, being massless, does not couple at tree level to SM fields [9. Darkphoton couplings to the SM fields can instead arise at one loop by means of higher-order operators, which are suppressed by the characteristic messenger mass scale.

In this framework, a new interesting phenomenology is expected that can be testable at the LHC [10, 11] and at future colliders [12]. For instance, Higgs effective couplings to photon $(\gamma)$ and dark photon $(\bar{\gamma})$, or to two dark photons, can arise at one loop due to the exchange of messenger and dark-fermion fields in [10]. These effective couplings can lead to exotic signatures, such as the one associated to the $H \rightarrow \gamma \bar{\gamma}$ decay, where the dark photon is observed in a detector as a massless invisible system. The LHC has an excellent potential to observe such decay for realistic branching ratios (BR's), in particular in the run 2 [10, 11]. Implications of the Higgs effective couplings to dark photons have also been analyzed in $e^{+} e^{-}$collisions [12].

In this paper we will focus on the dark-sector flavor structure needed to generate the CKM matrix in a theoretical framework based on the model in [4]. We will show that the required structure can potentially induce new exotic flavor-changing-neutral-current (FCNC) processes in the quark and lepton sectors. In particular, one foresees a new class of FCNC decay channels, namely the fermion decays to a lighter fermion of the same electric charge accompanied by a massless (invisible) dark photon,

$$
f \rightarrow f^{\prime} \bar{\gamma}
$$

We will first analyze the phenomenological implications of such FCNC decays in the topquark, bottom-quark, and charm-quark sectors, by studying the $t \rightarrow c \bar{\gamma}, u \bar{\gamma}, b \rightarrow s \bar{\gamma}, d \bar{\gamma}$, and $c \rightarrow u \bar{\gamma}$ decay channels, respectively. We will then extend the analysis to the leptonic sector, including the tau and muon decays $\tau \rightarrow \mu \bar{\gamma}, e \bar{\gamma}$, and $\mu \rightarrow e \bar{\gamma}$. In particular, we will compute different BR's and discuss their corresponding upper bounds coming from present phenomenological and theoretical constraints. 
As mentioned above, massless dark photons are decoupled at tree level from SM fields, and their production at colliders manifests as missing energy $\mathbb{E}$ and momentum $\not p$ in the detector, satisfying the kinematical neutrinolike constrain $\mathbb{E}^{2}-\not p^{2}=0$. As a consequence, the FCNC $f \rightarrow f^{\prime} \bar{\gamma}$ decay is characterized by an exotic experimental signature, where the final same-charge fermion $f^{\prime}$ is balanced in a detector by an invisible system with vanishing invariant mass. In the $f$ rest frame, neglecting radiative effects, $f^{\prime}$ is monochromatic with energy $E_{f^{\prime}} \simeq m_{f} / 2$, which is a very distinctive feature that would crucially discriminate $f \rightarrow f^{\prime} \bar{\gamma}$ backgrounds, where the missing momentum is associated either to the mismeasurement of hadronic objects or to the presence of nonmonochromatic neutrinos in the final states (as occurs in the $\mu$ or $\tau$ decays). Altogether a $f \rightarrow f^{\prime} \bar{\gamma}$ decay would show up experimentally by an excellent characterization.

The plan of the paper is the following. In Sec. 2, we present the theoretical framework, and provide the relevant Feynman rules for the computation of the FCNC $f \rightarrow f^{\prime} \bar{\gamma}$ decay amplitudes. In Sec. 3, we give the analytic expressions for the amplitude of a generic $f \rightarrow f^{\prime} \bar{\gamma}$ decay, and corresponding BR. In Sec. 4, 5, 6, 7, and 8, we will analyze the phenomenological implications for the FCNC decays in the top-quark, bottom-quark, charm-quark, $\tau$ and $\mu$ sectors, respectively. Our conclusions will be given in Sec. 9 .

\section{Theoretical framework}

In this section we summarize the main aspects of the flavor model in 4, providing the relevant interaction terms for the FCNC $f \rightarrow f^{\prime} \bar{\gamma}$ decays in the Lagrangian, and corresponding notation. More details on the model can be found in [4, 12].

As mentioned, the model extends the SM theory in order to generate radiatively Yukawa couplings at one loop, assuming vanishing tree-level Yukawa couplings. The corresponding total Lagrangian is made up of three sectors

$$
\mathcal{L}=\mathcal{L}_{S M}^{Y=0}+\mathcal{L}_{D S}+\mathcal{L}_{M S}
$$

where $\mathcal{L}_{S M}^{Y=0}$ is the SM Lagrangian for vanishing tree-level Yukawa couplings, $\mathcal{L}_{D S}$ is the darksector (DS) Lagrangian, containing the dark-fermion interactions with the $U(1)_{F}$ dark-photon gauge field, and $\mathcal{L}_{M S}$ describes the messenger sector with its couplings to both SM and dark fields. The $\mathcal{L}_{M S}$ interactions also communicate the ChSB and flavor structure of the dark sector to the observable SM sector, through the generation of Yukawa couplings at one loop.

\subsection{The dark-quark sector}

We start by recalling the $\mathcal{L}_{D S}$ Lagrangian related to the dark fermions associated to quarks (which we call dark quarks) and their interactions with the $U(1)_{F}$ gauge sector, including the mechanism to generate exponentially spread fermion masses. Its generalization to the leptonic sector will then be straightforward.

For each SM quark $q^{U_{i}, D_{i}}$ (with $i$ a family index), a quark replica $Q^{U_{i}, D_{i}}$ is assumed in the dark sector, which is singlet under SM gauge interactions, and charged under an exact $U(1)_{F}$ 
gauge symmetry. The corresponding Lagrangian is given by

$$
\begin{aligned}
\mathcal{L}_{D S} & =i \sum_{i}\left(\bar{Q}^{U_{i}} \mathcal{D}_{\mu} \gamma^{\mu} Q^{U_{i}}+\bar{Q}^{D_{i}} \mathcal{D}_{\mu} \gamma^{\mu} Q^{D_{i}}\right) \\
& -\frac{1}{4} F_{\mu \nu} F^{\mu \nu}+\frac{1}{2 \Lambda^{2}} \partial^{\mu} F_{\mu \alpha} \partial_{\nu} F^{\nu \alpha}
\end{aligned}
$$

where $\mathcal{D}_{\mu}=\partial_{\mu}+i g \hat{Q} A_{\mu}$ is the usual covariant derivative associated to the $U(1)_{F}$ dark-photon $A_{\mu}$ gauge field, with $\hat{Q}$ the corresponding charge operator acting on the $Q^{U_{i}}$ and $Q^{D_{i}}$ quark fields, and $F_{\mu \alpha}$ the $U(1)_{F}$ field-strength tensor. The higher-derivative last term in Eq. (5) is the so-called Lee-Wick term, where $\Lambda$ is the associated energy scale.

As shown in [13], because of the Lee-Wick term, which implies a massive spin-1 ghost particle in the spectrum, chiral symmetry turns out to be spontaneously broken, and dark fermions acquire mass nonperturbatively. In particular, by following the Nambu-Jona-Lasinio approach, one can show that a Dirac quark mass $M_{Q_{f}}$, solution of the fermion mass-gap equation corresponding to the true vacuum of the theory, exists in the weakly coupled regime in the form [13]

$$
M_{Q_{f}}=\Lambda \exp \left\{-\frac{2 \pi}{3 \bar{\alpha}(\Lambda) \bar{e}_{f}^{2}}+\frac{1}{4}\right\},
$$

where $\bar{e}_{f}$ stands for the $U(1)_{F}$ charge eigenvalue of a generic dark quark of flavor $f, Q_{f}$, in unit of the fundamental charge $\bar{e}$, and $\bar{\alpha}(\Lambda)$ is the effective fine structure constant (associated to $\bar{e}$ ) evaluated at the scale $\Lambda$. As already stressed, this solution is truly nonperturbative (as shown by the $\bar{\alpha}$ dependence in the exponent), and is associated to the true (nonperturbative) vacuum of the theory. For $N_{F}$ dark quarks with $\bar{e}_{f}$ charges $\left(f=1, \ldots N_{F}\right)$, an exponentially spread $M_{Q_{f}}$ spectrum can be generated by Eq. (6), just by requiring nonuniversality among the corresponding $\bar{e}_{f}$ charges. Indeed, since the $M_{Q_{f}}$ hierarchy in Eq. (6) will reflect into the actual SM fermion Yukawa hierarchy (as discussed in the following), it turns out that, for an integer sequence of $\bar{e}_{f}$ charges (and extending the present analysis to include the leptonic sector), one can easily fit most of the SM fermion mass spectrum [4].

\subsection{The messenger sector and the generation of Yukawa couplings}

The $\mathcal{L}_{M S}$ Lagrangian in Eq. (4) contains messenger scalar fields, and can be split in two terms

$$
\mathcal{L}_{M S}=\mathcal{L}_{M S}^{0}+\mathcal{L}_{M S}^{\mathrm{I}} \cdot
$$

$\mathcal{L}_{M S}^{0}$ includes the kinetic term for the messenger fields interacting with the SM gauge fields, while $\mathcal{L}_{M S}^{\mathrm{I}}$ provides the messenger interactions with the SM fermions, the dark fermions, and the Higgs boson, which are responsible for generating Yukawa couplings radiatively.

The SM quark gauge quantum numbers fix the minimal matter content needed for the colored messenger scalar sector, which is given by

- $2 N$ complex scalar $S U(2)_{L}$ doublets: $\hat{S}_{L}^{U_{i}}$ and $\hat{S}_{L}^{D_{i}}$, 


\begin{tabular}{|c||c|c|c|c|c|}
\hline Fields & Spin & $S U(2)_{L}$ & $U(1)_{Y}$ & $S U(3)_{c}$ & $U(1)_{F}$ \\
\hline$\hat{S}_{L}^{D_{i}}$ & 0 & $1 / 2$ & $1 / 3$ & 3 & $-\bar{e}_{D_{i}}$ \\
\hline$\hat{S}_{L}^{U_{i}}$ & 0 & $1 / 2$ & $1 / 3$ & 3 & $-\bar{e}_{U_{i}}$ \\
\hline$S_{R}^{D_{i}}$ & 0 & 0 & $-2 / 3$ & 3 & $-\bar{e}_{D_{i}}$ \\
\hline$S_{R}^{U_{i}}$ & 0 & 0 & $4 / 3$ & 3 & $-\bar{e}_{U_{i}}$ \\
\hline$Q^{D_{i}}$ & $1 / 2$ & 0 & 0 & 0 & $\bar{e}_{D_{i}}$ \\
\hline$Q^{U_{i}}$ & $1 / 2$ & 0 & 0 & 0 & $\bar{e}_{U_{i}}$ \\
\hline$S_{0}$ & 0 & 0 & 0 & 0 & 0 \\
\hline
\end{tabular}

Table 1: Spin and gauge quantum numbers for the strongly interacting messenger fields and corresponding dark quarks. $U(1)_{F}$ is the dark-photon gauge symmetry in the dark sector.

- $2 N$ complex scalar $S U(2)_{L}$ singlets: $S_{R}^{U_{i}}$ and $S_{R}^{D_{i}}$,

- one real $S U(2)_{L} \times U(1)_{Y}$ singlet: $S_{0}$,

where $\hat{S}_{L}^{U_{i}, D_{i}}=\left(\begin{array}{c}S_{L, 1}^{U_{i}, D_{i}} \\ S_{L, 2}^{U_{i}, D_{i}}\end{array}\right)$, and $i=1, \ldots, N(N=3)$ stands for a family index. The $\hat{S}_{L}^{U_{i}, D_{i}}$, $S_{R}^{U_{i}, D_{i}}$ scalar fields have the SM quark quantum numbers, where the $L, R$ labels identify the messengers coupled to the $L, R$ chirality components of the associated SM quarks (just as occurs in the case of squark fields in supersymmetric theories). They have minimal gauge-invariant couplings to electroweak (EW) gauge bosons and gluons. A minimal flavor violation hypothesis would require the Lagrangian in Eq. (7) to be invariant under $S U\left(N_{F}\right)$, where $N_{F}$ is the number of flavors. More generally, for any family index $i$, we can reduce the messenger mass sector to four different universal mass terms corresponding to the up/down and $L / R$ components of the $\hat{S}_{L, R}^{U_{i}}$ and $\hat{S}_{L, R}^{D_{i}}$ sectors, as in minimal supersymmetric models. Notice that a more minimal hypothesis of a common scalar mass for the $L$ and $R$ scalar sectors is also phenomenologically acceptable.

We do not report here the expression for the interaction Lagrangian of the messenger fields with the SM gauge bosons, which follows from the universal properties of gauge interactions. Notice that each messenger field is also charged under $U(1)_{F}$, and carries the same $U(1)_{F}$ charge of the associated dark fermion. In other words, $U(1)_{F}$ charges identify the flavor state. A summary of relevant quantum numbers for all new fermion and scalar fields in the quark sector can be found in Table 1 .

The $\mathcal{L}_{M S}^{I}$ Lagrangian, which describes the messenger interactions with quarks and SM Higgs boson, is particularly relevant for the SM flavor structure. The minimal content of the universal 
interactions needed to generate radiative (diagonal) Yukawa couplings is

$$
\begin{aligned}
\mathcal{L}_{M S}^{I} & =\left\{g_{L}\left(\sum_{i=1}^{N}\left[\bar{q}_{L}^{i} Q_{R}^{U_{i}}\right] \hat{S}_{L}^{U_{i}}+\sum_{i=1}^{N}\left[\bar{q}_{L}^{i} Q_{R}^{D_{i}}\right] \hat{S}_{L}^{D_{i}}\right)\right. \\
& +g_{R}\left(\sum_{i=1}^{N}\left[\bar{U}_{R}^{i} Q_{L}^{U_{i}}\right] S_{R}^{U_{i}}+\sum_{i=1}^{N}\left[\bar{D}_{R}^{i} Q_{L}^{D_{i}}\right] S_{R}^{D_{i}}\right) \\
& \left.+\lambda_{S} S_{0} \sum_{i=1}^{N}\left(\tilde{H}^{\dagger} S_{L}^{U_{i}} S_{R}^{U_{i} \dagger}+H^{\dagger} S_{L}^{D_{i}} S_{R}^{D_{i} \dagger}\right)+H . c .\right\}+V\left(S_{0}\right),
\end{aligned}
$$

where contractions with color indices are understood. The $S_{0}$ field is a real singlet scalar, and its potential $V\left(S_{0}\right)$ is invariant under the $S_{0} \rightarrow-S_{0}$ parity symmetry. The $g_{L}$ and $g_{R}$ constants are flavor-universal free parameters, whose values can be in the perturbative regime $g_{L, R} \lesssim 1$. We will assume in general $g_{L} \neq g_{R}$, although one could impose a higher degree of universality by assuming $g_{L}=g_{R}$, with no loss of generality in the prediction of Yukawa couplings. In Eq. (8), $q_{L}^{i}, U_{R}^{i}$, and $D_{R}^{i}$ stand for SM quark fields, and $H$ is the SM Higgs doublet, with $\tilde{H}=i \sigma_{2} H^{\star}$. One can then prevent Yukawa couplings at tree level by imposing a combined parity symmetry under $H \rightarrow-H$ and $S_{0} \rightarrow-S_{0}$.

On the other hand, as shown in [4], after spontaneous symmetry breaking (SSB) of the $H \rightarrow-H$ and $S_{0} \rightarrow-S_{0}$ parity symmetry by a nonvanishing vacuum expectation value (VEV) $\left\langle S_{0}\right\rangle \equiv \mu_{S} / \lambda_{S}$, the Yukawa couplings can be radiatively generated at one loop via virtual exchange of messengers and dark fermions. As a result, the effective Yukawa coupling associated to the quark of flavor $f$ turns out to be proportional to the corresponding dark-quark mass $M_{Q_{f}}$. In particular, one obtains [4]

$$
Y_{f}=Y_{0}\left(x_{f}\right) \exp \left(-\frac{2 \pi}{3 \bar{\alpha}(\Lambda) \bar{e}_{f}^{2}}\right),
$$

where the dark-quark mass $M_{Q_{f}}$ has been replaced by Eq. (6), the one-loop $Y_{0}\left(x_{f}\right)$ function is given by

$$
Y_{0}\left(x_{f}\right)=\left(\frac{g_{L} g_{R}}{16 \pi^{2}}\right)\left(\frac{\mu_{S} \Lambda}{\bar{m}^{2}}\right) C_{0}\left(x_{f}\right)
$$

$\bar{m}^{2}$ is the mean square mass of the messengers running in the loop, $x_{f}=M_{Q_{f}}^{2} / \bar{m}^{2}$, and

$$
C_{0}(x)=\frac{1-x(1-\log x)}{(1-x)^{2}}
$$

Equation (10) is obtained in the approximation of degenerate messenger masses for generic $S U(2)_{L}$ doublet $S_{L}$ and singlet $S_{R}$ fields, and in the limit of small mixing parameter $\xi=\Delta / \bar{m}^{2}$, with $\Delta=\mu_{S} v$, and $v$ the Higgs VEV.

As from Eqs.(9) and (10), the top-quark Yukawa coupling can be large and $\mathcal{O}(1)$, keeping at the same time the dimensionless couplings $g_{L}, g_{R}$ small and within the perturbative regime. 
Indeed the Yukawa coupling turns out to be proportional to the singlet-field $(S) \operatorname{VEV}\left(\mu_{S} / \lambda_{S}\right)$, and is generated only after the spontaneous breaking of the $Z_{2}$ symmetry. This is a general property, which is independent from the particular symmetry forbidding Yukawa couplings at the tree level. Then, a $\mathcal{O}(1)$ Yukawa coupling can be achieved by choosing the $\mu_{S}$ scale larger than the characteristic $\bar{m}$-mass scale running in the loop, while keeping all other dimensionless couplings small and in the perturbative range.

In order to extend the above results to larger $\xi$ mixing values, one can use the mass-eigenstate basis for messengers. Notice that, after the EW symmetry breaking, terms in the third row of the Lagrangian in Eq.(8) generate a mixing term $\Delta$ between the $S U(2)_{L}$ messenger doublet $S_{L}$, and the corresponding singlet $S_{R}$. The corresponding Lagrangian for generic $S_{L, R}$ fields is

$$
\mathcal{L}_{S}^{0}=\partial_{\mu} \hat{S}^{\dagger} \partial^{\mu} \hat{S}-\hat{S}^{\dagger} \hat{M}_{S}^{2} \hat{S}
$$

where $\hat{S}=\left(S_{L}, S_{R}\right)$, and the mass term involves the mass matrix

$$
\hat{M}_{S}^{2}=\left(\begin{array}{cc}
m_{L}^{2} & \Delta \\
\Delta & m_{R}^{2}
\end{array}\right)
$$

with $\Delta=\mu_{S} v$ parametrizing the left-right $(L R)$ scalar mixing. The $\hat{M}_{S}^{2}$ matrix in Eq. 13 can be diagonalized by the unitary matrix

$$
U=\left(\begin{array}{cc}
\cos \theta & \sin \theta \\
-\sin \theta & \cos \theta
\end{array}\right)
$$

with $\tan 2 \theta=\frac{2 \Delta}{m_{L}^{2}-m_{R}^{2}}$. Then, the eigenvalues of the diagonal matrix $\hat{M}_{S}^{2 \operatorname{diag}}=U \hat{M}_{S}^{2} U^{\dagger}$ are given by

$$
m_{ \pm}^{2}=\frac{1}{2}\left(m_{L}^{2}+m_{R}^{2} \pm\left[\left(m_{L}^{2}-m_{R}^{2}\right)^{2}+4 \Delta^{2}\right]^{1 / 2}\right)
$$

where, for degenerate $L R$ scenarios (namely for $m_{L}^{2}=m_{R}^{2}=\bar{m}^{2}$ ), the $U$ matrix elements simplify to $U(i, i)=1 / \sqrt{2}, U(1,2)=-U(2,1)=1 / \sqrt{2}$, with square mass eigenvalues

$$
m_{ \pm}^{2}=\bar{m}^{2}(1 \pm \xi), \quad \xi=\Delta / \bar{m}^{2}
$$

Note that, in order to prevent tachyonic solutions, one should impose $\xi \leq 1$ with $\Delta>0$. Then, we computed the generalization of the $Y_{0}$ expression in Eq. 10 as a function of $\xi$, in the degenerate $L R$ scenario, which turns out to be

$$
Y_{0}\left(x_{f}, \xi\right)=\left(\frac{g_{L} g_{R}}{16 \pi^{2}}\right)\left(\frac{\xi \Lambda}{v}\right) f_{1}\left(x_{f}, \xi\right),
$$

where

$$
f_{1}(x, \xi)=\frac{1}{2}\left[C_{0}\left(\frac{x}{1-\xi}\right) \frac{1}{1-\xi}+C_{0}\left(\frac{x}{1+\xi}\right) \frac{1}{1+\xi}\right]
$$

and $C_{0}(x)$ is defined by Eq. (11). Notice that $C_{0}(1)=1 / 2$, and, for small $x \ll 1, C_{0}(x) \simeq$ $1+\mathcal{O}(x)$. Indeed, at fixed values of $\bar{m}$ and $\Lambda$, all Yukawa couplings must vanish for vanishing mixing $\xi \rightarrow 0$, since they are proportional to the VEV of the singlet field $S, \mu_{S}$ (cf. Eq. (10)). 


\subsection{The flavor structure and the CKM matrix}

Although predicting exponentially spread Yukawa couplings and providing a natural solution to the flavor hierarchy problem, the minimal interaction Lagrangian $\mathcal{L}_{M S}^{I}$ in Eq.(8) does not account for the observed CKM mixing matrix of weak interactions. Indeed, the radiatively generated Yukawa couplings turn out to be diagonal in the weak-current interaction basis for the quark fields. Yukawa off diagonal terms are needed to generate the CKM, and, in order to preserve the $U(1)_{F}$ gauge invariance, the universal flavor structure of the messenger interaction in Eq.(8) should be generalized as follows:

$$
\begin{aligned}
\tilde{\mathcal{L}}_{M S}^{I} & =\left\{g_{L}\left(\sum_{i, j=1}^{N}\left[\bar{q}_{L}^{i}\left(X_{L}^{U}\right)_{i j} Q_{R}^{U_{j}}\right] \hat{S}_{L}^{U_{j}}+\sum_{i, j=1}^{N}\left[\bar{q}_{L}^{i}\left(X_{L}^{D}\right)_{i j} Q_{R}^{D_{j}}\right] \hat{S}_{L}^{D_{j}}\right)\right. \\
& +g_{R}\left(\sum_{i, j=1}^{N}\left[\bar{U}_{R}^{i}\left(X_{R}^{U}\right)_{i j} Q_{L}^{U_{j}}\right] S_{R}^{U_{j}}+\sum_{i, j=1}^{N}\left[\bar{D}_{R}^{i}\left(X_{L}^{D}\right)_{i j} Q_{L}^{D_{j}}\right] S_{R}^{D_{j}}\right) \\
& \left.+\lambda_{S} S_{0} \sum_{i=1}^{N}\left(\tilde{H}^{\dagger} S_{L}^{U_{i}} S_{R}^{U_{i} \dagger}+H^{\dagger} S_{L}^{D_{i}} S_{R}^{D_{i} \dagger}\right)+H . c .\right\}+V\left(S_{0}\right),
\end{aligned}
$$

where $X_{L, R}^{U, D}$ are generic (not necessarily unitary) matrices. Notice that the $U(1)_{F}$ gauge invariance and nonuniversality of $U(1)_{F}$ charges require the family index labeling dark fermions and scalar messengers to be the same. Then, in the weak-current basis for quark fields, the Yukawa couplings generated radiatively follow the pattern

$$
Y_{i j}^{U, D} \sim\left(X_{L}^{U, D \dagger} \cdot \hat{Y}^{U, D} \cdot X_{R}^{U, D}\right)_{i j}
$$

where the - symbol stands for a matrix product, and $\hat{Y}^{U, D}=\operatorname{diag}\left[Y_{1}^{U, D}, Y_{2}^{U, D}, Y_{3}^{U, D}\right]$, with $Y_{i}^{U, D}(i=1,2,3)$ standing for the Yukawa couplings in Eq. $(9)$ for the up and down sectors. Following the usual SM approach, the Yukawa matrix in Eq. 20 can be diagonalized by a biunitary rotation $V_{L, R}^{U, D}$, namely

$$
\operatorname{diag}\left[Y^{U, D}\right]=V_{L, R}^{U, D} \dagger \cdot Y^{U, D} \cdot V_{L, R}^{U, D}
$$

hence giving rise to the CKM matrix $K=V_{L}^{U \dagger} \cdot V_{L}^{D}$.

The observed structure of the CKM matrix requires $X_{L, R}^{U, D}$ to have off diagonal entries smaller than the diagonal ones, with the latter almost proportional to the unity matrix $\mathbf{1}$ in the family space. This suggests the following ansatz for the $X_{L, R}^{U, D}$ matrices

$$
X_{L, R}^{U, D} \sim \mathbf{1}+\Delta_{L, R}^{U, D}
$$

where the matrices $\left|\Delta_{L, R}^{U, D}\right| \ll 1$ collect diagonal and off diagonal terms in a less hierarchical structure 2 .

\footnotetext{
${ }^{2}$ We suggest a possible renormalization mechanism for generating a flavor structure of the $X_{L, R}^{U, D}$ matrices as required by Eq. 22, assuming universal tree-level couplings like in Eq. 8). This requires new heavy (either
} 
After rotating the quark fields to the basis of mass eigenstates (entailing diagonal Yukawa couplings), the interaction terms in Eq. 19 can be transformed by replacing the $X_{L, R}^{U, D}$ matrices according to

$$
X_{L, R}^{U} \rightarrow \rho_{L, R}, \quad X_{L, R}^{D} \rightarrow \eta_{L, R},
$$

where

$$
\begin{aligned}
\rho_{L, R} & \equiv V_{L, R}^{U \dagger} \cdot X_{L, R}^{U}, \\
\eta_{L, R} & \equiv V_{L, R}^{D \dagger} \cdot X_{L, R}^{D} .
\end{aligned}
$$

If $X_{L}^{U, D}$ are unitary matrices, then $V_{L, R}^{U, D}=\left(X_{L}^{U, D}\right)^{-1}$, the $\rho_{L, R}, \eta_{L, R}$ matrices will just be equal to $\mathbf{1}$, and the CKM matrix will be $K=X_{L}^{U} \cdot X_{L}^{D \dagger}$. However, $X_{L}^{U, D}$ matrices do not need to be unitary (or proportional to a unitary matrix), since they do not arise from unitary transformations (like, e.g., in the CKM-matrix case). In general, $\rho_{L, R}$ and $\eta_{L, R}$ will then have nonvanishing off diagonal entries. This has nontrivial consequences, since off diagonal terms in the $\rho_{L, R}$ and $\eta_{L, R}$ matrices can induce FCNC interactions at one loop in the observable quark and lepton sectors.

Among the FCNC processes induced by these new interactions, there is a new class of FCNC one-loop decays, that is SM-fermion decays into a massless dark photon, via the channels $q \rightarrow q^{\prime} \bar{\gamma}\left(\ell \rightarrow \ell^{\prime} \bar{\gamma}\right)$, where $q^{\prime}\left(\ell^{\prime}\right)$ is a lighter quark (lepton) with same charge as $q(\ell)$.

In the next section, we will compute the relevant amplitudes and corresponding decay widths for this new class of processes, as well as the NP contribution to the $q \rightarrow q^{\prime} \gamma$ and $\ell \rightarrow \ell^{\prime} \gamma$ decays into a SM photon. The Feynman rules relevant for the computation of the $q \rightarrow q^{\prime} \bar{\gamma}$ decay amplitude (with straightforward extension to the leptonic sector) can be found in Fig. 1.

\section{$3 \quad$ The $q \rightarrow q^{\prime} \bar{\gamma}$ amplitude and decay width}

For a generic quark $q^{i}$, with $q=U, D$, we consider the FCNC decay process

$$
q^{i}(p) \rightarrow q^{j}\left(p^{\prime}\right) \bar{\gamma}(k)
$$

where the indices $i, j$ run over quark families with $i>j$, and $p, p^{\prime}$, and $k$ indicate the particle four-momenta. A generalization to the leptonic sector is straightforward. This process is induced at one loop by the Lagrangian in Eq. (19) for quarks (and by its leptonic generalization for lepton decays).

The Feynman diagrams contributing to the $q^{i} \rightarrow q^{j} \bar{\gamma}$ process are given in Fig. 2. There are

scalar or vector) fields in the dark sector, which are SM gauge singlets, and are charged under $U(1)_{F}$ with charges $Q_{i j}=\bar{e}_{i}-\bar{e}_{j}(i, j=1,2,3)$. Gauge invariant couplings of these new fields to both dark fermions and messenger scalars can be formed. One-loop corrections to the vertices of the universal interactions in Eq. 8), induced by these new interactions in the dark sector, can then generate the desired off diagonal transitions that can be reabsorbed in the matrix elements $\Delta_{i j}$. Being $\Delta_{i j}$ generated at higher orders in perturbation theory, the hierarchy shown in Eq. (22) is automatically satisfied. We will not consider this possibility here, and will assume the most general structure for the $X$ matrices, no matter what mechanism has generated them. 

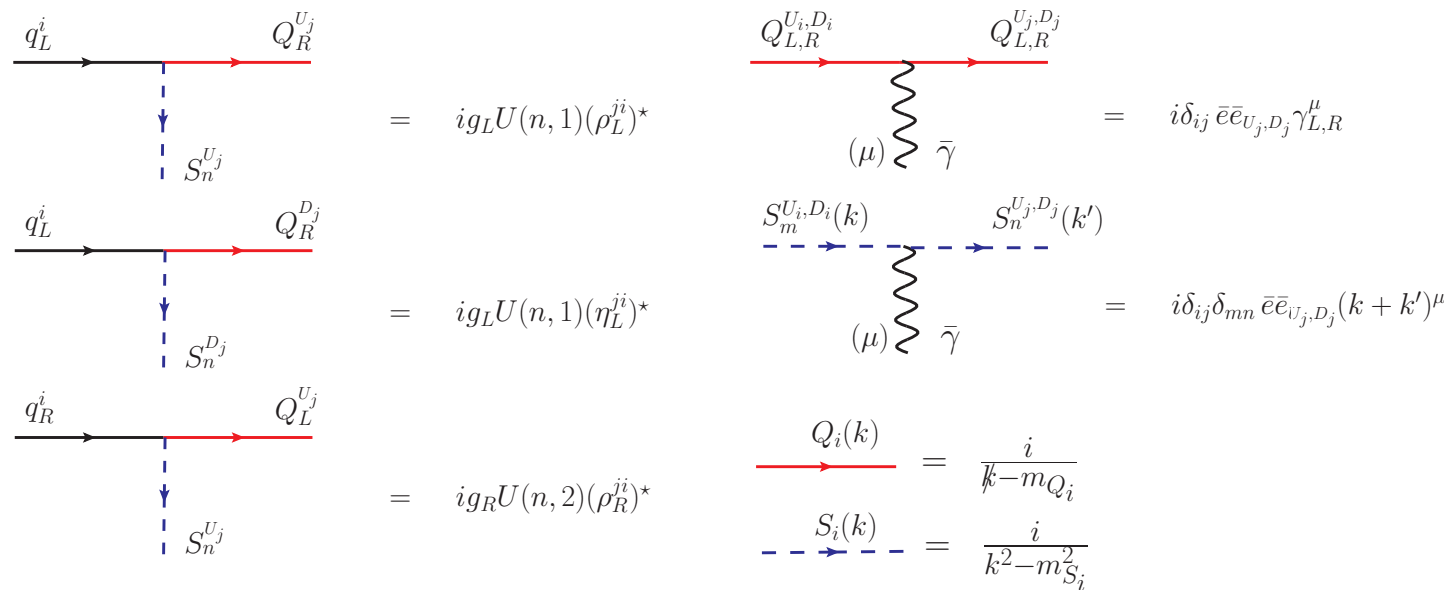

Figure 1: Feynman rules for interaction vertices and propagators entering the computation of the one-loop $q \rightarrow q^{\prime} \bar{\gamma}$ decay amplitude. The symbols $q_{L / R}^{i}$ and $Q_{L / R}^{U_{i}, D_{i}}$ stand for the quark and dark-quark fields, respectively, with $L / R$ denoting the left-/right-handed chirality projections. $S_{n}^{U_{j}}$ and $S_{n}^{D_{j}}$ stand for the mass eigenstates $(n=1,2)$ in the up and down messenger sectors, respectively, while $\bar{\gamma}$ is the dark-photon field.

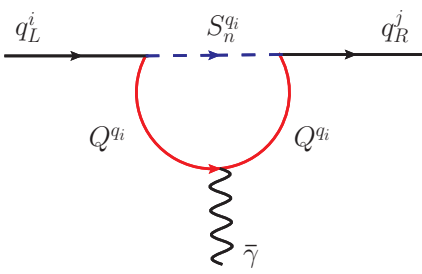

(a)

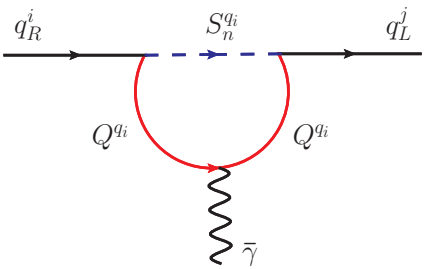

(c)

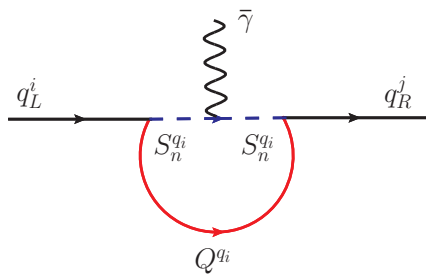

(b)

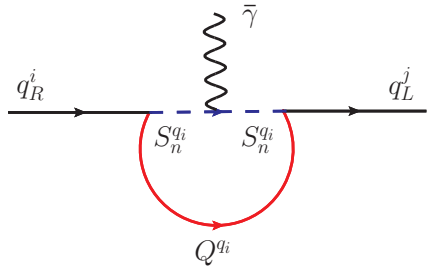

$(d)$

Figure 2: Feynman diagrams (a)-(d) contributing to the FCNC decay $q^{i} \rightarrow q^{j} \bar{\gamma}$ with $q=U, D$ and $i>j$, where $q_{L, R}^{i, j}$ are the initial $(i)$, final $(j)$ quarks, with $L / R$ indicating the left/right chirality projections, $Q^{q_{i}}$ and $S_{n}^{q_{i}}$ the corresponding dark quarks and messenger fields, respectively, with the latter in the basis of mass eigenstates $(n=1,2)$, while $\bar{\gamma}$ stands for the dark-photon line. 
no self-energy contributions to the $q^{i} \rightarrow q^{j} \bar{\gamma}$ process, since the dark photon does not couple to SM fermions at tree level. The messengers running in the loop are much heavier than the external fermion states (also in case of the top-quark decay), and we can safely neglect terms of order $\mathcal{O}\left(m_{q_{i}}^{2} / \bar{m}^{2}\right)$, where $m_{q_{i}}$ are the external-quark masses. However, we will retain the leading contributions induced by the initial SM fermion mass, or, equivalently, by its associated Yukawa coupling, and neglect the contributions of the final quark mass.

The total amplitude in momentum space receives two independent gauge-invariant contributions

$$
M\left(q^{i} \rightarrow q^{j} \bar{\gamma}\right)=M\left(q_{L}^{i} \rightarrow q_{R}^{j} \bar{\gamma}\right)+M\left(q_{R}^{i} \rightarrow q_{L}^{j} \bar{\gamma}\right)
$$

where $q_{L / R}^{i}$ are chirality eigenstates in the $q=U, D$ sectors. The two contributions can be parametrized as follows

$$
\begin{aligned}
M\left(q_{L}^{i} \rightarrow q_{R}^{j} \bar{\gamma}\right) & =\frac{1}{\left(\Lambda_{L}^{q}\right)_{i j}}\left[\bar{u}_{R}^{q_{j}} \sigma_{\alpha \mu} u_{L}^{q_{i}}\right] k^{\mu} \bar{\epsilon}^{\alpha}, \\
M\left(q_{R}^{i} \rightarrow q_{L}^{j} \bar{\gamma}\right) & =\frac{1}{\left(\Lambda_{R}^{q}\right)_{i j}}\left[\bar{u}_{L}^{q_{j}} \sigma_{\alpha \mu} u_{R}^{q_{i}}\right] k^{\mu} \bar{\epsilon}^{\alpha},
\end{aligned}
$$

where $\sigma_{\mu \nu} \equiv \frac{1}{2}\left[\gamma_{\mu}, \gamma_{\nu}\right]$ ([a,b] standing for the $a$ and $b$ matrix commutator $), u_{L / R} \equiv \frac{1}{2}\left(1 \mp \gamma_{5}\right) u$, and $u^{q_{i}}$ and $u^{q_{j}}$ correspond respectively to the $q^{i}$ and $q^{j}$ on shell bispinors in momentum space, $\bar{\epsilon}^{\alpha}$ being the dark-photon polarization vector. Gauge invariance requires $k_{\mu} \bar{\epsilon}^{\mu}=0$ for on shell dark photons, which makes the contribution proportional to the $\gamma_{L, R}^{\mu}$ matrices vanish for on shell massless (i.e., for $k^{2}=0$ ) dark photons. As a consistency check, we have controlled that this condition is satisfied at one loop.

Then, the effective scales associated to the matrix elements $\left(\Lambda_{L, R}^{q}\right)_{i j}$ can be derived by matching Eq. (28) with the computation of the transition amplitude, based on the Feynman diagrams in Fig. 2. We assume massless final fermions, which will be a proper approximation for the processes considered in the following.

The contribution to the magnetic-dipole type of operator $\left[\bar{u}_{R}^{q_{j}} \sigma_{\alpha \mu} u_{L}^{q_{i}}\right]$ (which is finite and $U(1)_{F}$ gauge invariant) involves different chiralities in the external $u^{q_{i}}$ and $\bar{u}^{q_{j}}$ states. There are two different sources for the chirality flip. One corresponds to the mass insertion of a virtual dark quark through its propagator, and the second arises from the external quark masses, after the on shell conditions on $u^{q_{i}}$ or $\bar{u}^{q_{j}}$ are applied. Since we are assuming massless final fermions, only initial fermions contribute to the latter.

Finally, after some algebraic manipulations, the $\Lambda_{L, R}^{U}$ scales relative to a generic FCNC transition $q^{i} \rightarrow q^{j}$, with $q$ in the $u p$ fermion sector, and $i, j(i>j)$ running over three fermion generations, become

$$
\begin{aligned}
\frac{1}{\left(\Lambda_{L}^{U}\right)_{i j}} & =\frac{\bar{e} m_{U_{i}}}{\bar{m}_{U}^{2}}\left[\bar{e}_{i}^{U} \frac{\rho_{R}^{j i}}{\rho_{R}^{i i}} F_{L R}\left(x_{i}^{U}, \xi_{U}\right)-\frac{g_{R}^{2}}{16 \pi^{2}} \sum_{k=1}^{3} \bar{e}_{k}^{U} \rho_{R}^{j k} \rho_{R}^{k i} F_{R R}\left(x_{k}^{U}, \xi_{U}\right)\right] \\
\frac{1}{\left(\Lambda_{R}^{U}\right)_{i j}} & =\frac{\bar{e} m_{U_{i}}}{\bar{m}_{U}^{2}}\left[\bar{e}_{i}^{U} \frac{\rho_{L}^{j i}}{\rho_{L}^{i i}} F_{R L}\left(x_{i}^{U}, \xi_{U}\right)-\frac{g_{L}^{2}}{16 \pi^{2}} \sum_{k=1}^{3}\left(\bar{e}_{k}^{U} \rho_{L}^{j k} \rho_{L}^{k i} F_{L L}\left(x_{k}^{U}, \xi_{U}\right)\right.\right. \\
& \left.\left.+\left(\frac{\bar{m}_{U}^{2}}{\bar{m}_{D}^{2}}\right) \bar{e}_{k}^{D} \eta_{L}^{j k} \eta_{L}^{k i} F_{L L}\left(x_{k}^{D}, \xi_{D}\right)\right)\right]
\end{aligned}
$$


being $\bar{e}$ the charge unit for dark-photon interactions, $\bar{e}_{k}^{q}$ their eigenvalues, $m_{U_{i}, D_{i}}$ the initialquark masses, $M_{Q_{i}^{U, D}}$ the corresponding dark-quark masses, $x_{i}^{U, D} \equiv M_{Q_{i}^{U, D}}^{2} / \bar{m}_{U, D}^{2}$, and $\bar{m}_{U, D}$, $\xi_{U, D}$, respectively, the common average mass and mixing parameter in the up, down messenger sectors.

For $\Lambda_{L, R}^{D}$ in the down quark sector, we obtain instead

$$
\begin{aligned}
\frac{1}{\left(\Lambda_{L}^{D}\right)_{i j}} & =\frac{\bar{e} m_{D_{i}}}{\bar{m}_{D}^{2}}\left[\bar{e}_{i}^{D} \frac{\eta_{R}^{j i}}{\eta_{R}^{i i}} F_{L R}\left(x_{i}^{D}, \xi_{D}\right)-\frac{g_{R}^{2}}{16 \pi^{2}} \sum_{k=1}^{3} \bar{e}_{k}^{D} \eta_{R}^{j k} \eta_{R}^{k i} F_{R R}\left(x_{k}^{D}, \xi_{D}\right)\right] \\
\frac{1}{\left(\Lambda_{R}^{D}\right)_{i j}} & =\frac{\bar{e} m_{D_{i}}}{\bar{m}_{D}^{2}}\left[\bar{e}_{i}^{D} \frac{\eta_{L}^{j i}}{\eta_{L}^{i i}} F_{R L}\left(x_{i}^{D}, \xi_{D}\right)-\frac{g_{L}^{2}}{16 \pi^{2}} \sum_{k=1}^{3}\left(\bar{e}_{k}^{D} \eta_{L}^{j k} \eta_{L}^{k i} F_{L L}\left(x_{k}^{D}, \xi_{D}\right)\right.\right. \\
& \left.\left.+\left(\frac{\bar{m}_{D}^{2}}{\bar{m}_{U}^{2}}\right) \bar{e}_{k}^{U} \rho_{L}^{j k} \rho_{L}^{k i} F_{L L}\left(x_{k}^{U}, \xi_{U}\right)\right)\right]
\end{aligned}
$$

The first terms in the right-hand side of Eqs. $29-30$ for the effective $\Lambda_{L, R}^{D, U}$ scales are independent from the $g_{L, R}$ couplings, since this dependence has been reabsorbed in the corresponding SM Yukawa couplings, by using Eq. (17).

Furthermore, the loop functions appearing in Eqs. (29)-(30) satisfy the conditions $F_{R R}(x, \xi)=$ $F_{L L}(x, \xi)$, and $F_{R L}(x, \xi)=F_{L R}(x, \xi)$, where

$$
\begin{aligned}
& F_{L L}(x, \xi)=\frac{1}{8}\left[\frac{x^{2}-(\xi-1)^{2}+2 x(\xi-1) \log \left(\frac{x}{1-\xi}\right)}{(x-1+\xi)^{3}}+\{\xi \rightarrow-\xi\}\right], \\
& F_{L R}(x, \xi)=\frac{f_{2}(x, \xi)}{f_{1}(x, \xi)}
\end{aligned}
$$

defining

$$
f_{2}(x, \xi)=\frac{1}{2 \xi}\left[\frac{1-x+\xi+(1+\xi) \log \left(\frac{x}{1+\xi}\right)}{(1-x+\xi)^{2}}-\{\xi \rightarrow-\xi\}\right],
$$

and for $f_{1}(x, \xi)$ given by Eq.(18).

Some comments on Eqs. 29)-(30) are in order. Terms proportional to $F_{L R}$ and $F_{R L}$ arise from the chirality flip induced by the virtual-dark-fermion mass insertion. Terms proportional to $F_{L L}$ and $F_{R R}$ come instead from the chirality flip induced by the initial-quark mass $m_{U_{i}, D_{i}}$, after applying on shell relations on external momenta $\not p u_{L, R}^{f}(p)=m_{f} u_{R, L}^{f}(p)$, for a generic fermion $f$ of mass $m_{f}$. In the present model, all contributions turn out to be proportional to the initial quark mass. This is because the dark-quark mass insertion has been reabsorbed in the corresponding quark mass, by using the model prediction for the one-loop effective Yukawa coupling in Eq. (10). However, for $\bar{m}_{U}$ and $\bar{m}_{D}$ of the same order, terms proportional to $F_{L L / R R}$ are subleading with respect to the ones proportional to $F_{L R / R L}$, due to the suppression of the loop factors $\left(g_{L, R}^{2} / 16 \pi^{2}\right)$ in Eqs. (29)- 30 . 
Finally, we report some useful analytical expressions for $F_{L L}(x, \xi)$ and $F_{L R}(x, \xi)$ in the limit of small and large values of the mixing parameter $\xi$. For $\xi \ll 1$ one gets

$$
\begin{array}{ll}
\lim _{\xi \rightarrow 0} F_{L R}(x, \xi)=\frac{2(1-x)+(1+x) \ln x}{(x-1)(1-x+\ln x)}, & \lim _{\xi \rightarrow 0} F_{L L}(x, \xi)=\frac{x^{2}-1-2 x \ln x}{4(x-1)^{3}}, \\
\lim _{x \rightarrow 1} \lim _{\xi \rightarrow 0} F_{L R}(x, \xi)=-1 / 3 & \lim _{x \rightarrow 1} \lim _{\xi \rightarrow 0} F_{L L}(x, \xi)=1 / 12,
\end{array}
$$

while, for large mixing $\xi \sim 1$, we get ${ }^{3}$

$$
\begin{aligned}
F_{L R}(x, \xi) \simeq & \frac{x(2+\ln 4)-4-2 x \ln x}{4-6 x+x^{2}(2+\ln 2)+(x-2)^{2} \ln (1-\xi)-2\left(2-2 x+x^{2}\right) \ln x}+\mathcal{O}(1-\xi), \\
& \lim _{\xi \rightarrow 1} F_{L L}(x, \xi)=\frac{4(x-1)-3 x^{2}+x^{3}-2 x^{2} \ln \frac{x}{2}}{4(x-2)^{3} x} .
\end{aligned}
$$

Since messenger masses are expected to be quite heavy [4], the $q^{i} \rightarrow q^{j} \bar{\gamma}$ decay process can actually be described by an effective Lagrangian approach. The relevant effective density Lagrangian $\mathcal{L}_{\text {eff }}$ contains two leading gauge-invariant operators of dimension 5 , that is the FC magnetic-dipole operators given by

$$
\mathcal{L}_{\text {eff }}=\sum_{q=U, D} \sum_{i, j=1}^{3}\left(\frac{1}{2\left(\Lambda_{L}^{q}\right)_{i j}}\left[\bar{q}_{R}^{j}(x) \sigma_{\mu \nu} \bar{F}^{\mu \nu}(x) q_{L}^{i}(x)\right]+\frac{1}{2\left(\Lambda_{R}^{q}\right)_{i j}}\left[\bar{q}_{L}^{j}(x) \sigma_{\mu \nu} \bar{F}^{\mu \nu}(x) q_{R}^{i}(x)\right]\right),
$$

where $i>j, \bar{F}^{\mu \nu}(x)$ is the dark-photon $U(1)_{F}$ field-strength tensor, $q^{i}(x)$ and $q^{j}(x)$ are the initial and final quark fields, and $\Lambda_{L, R}^{U}$ and $\Lambda_{L, R}^{D}$ are given in Eqs. 29) and (30), respectively.

Using the effective Lagrangian in Eq. (38), the total width for $q^{2} \rightarrow q^{j} \bar{\gamma}$ is (neglecting the final quark mass)

$$
\Gamma\left(q^{i} \rightarrow q^{j} \bar{\gamma}\right)=\frac{m_{q_{i}}^{3}}{16 \pi^{3}}\left(\frac{1}{\left(\Lambda_{L}^{q}\right)_{i j}^{2}}+\frac{1}{\left(\Lambda_{R}^{q}\right)_{i j}^{2}}\right) .
$$

Notice that, due to the chiral suppression of the initial quark masses $m_{q_{i}}$ entering in the $\Lambda_{L, R}^{q}$ scales [see Eqs. 29]-(30)], the width turns out to be proportional to the fifth power of the decaying quark mass $m_{q_{i}}$, suppressed by the fourth power of the corresponding average messenger mass $\bar{m}_{q}$, according to the expression

$$
\left.\Gamma\left(q^{i} \rightarrow q^{j} \bar{\gamma}\right) \sim \frac{m_{q_{i}}^{5}}{16 \pi^{3} \bar{m}_{q}^{4}} \times \text { (loop functions }\right)
$$

In the following discussion, the relevant independent parameters will be $\bar{m}_{q}$ (which controls the order of magnitude of the decay width), the mixing parameter $\xi_{q}$, (which, at large values $\sim 1$,

\footnotetext{
${ }^{3}$ In order to avoid stable messenger particles in the spectrum, for a generic quark sector $q$, the corresponding mixing parameter $\xi$ should be bounded by $0<\xi<1-x$, where $x=m_{Q}^{2} / \bar{m}^{2}$, and $m_{Q}$ is the associated dark-fermion mass (see next section). Then, the logarithmic term $\ln (1-\xi)$, appearing in the $F_{L R}$ denominator in the large $\xi \rightarrow 1$ expansion [see Eq. [36)], will be bounded by $\ln (1-\xi)<\ln x$. Since $x$ is nonvanishing (being dark fermions heavier than the corresponding SM fermions), $F_{L R}(x, \xi)$ and $F_{L L}(x, \xi)$ will not develop any singularity in the allowed $x$ and $\xi$ ranges.
} 
pushes the smallest $\bar{m}_{q}$ eigenvalues of the messengers running in the loop toward the lowest values $\left[c f\right.$. Eq. (16)], hence enhancing the decay amplitudes), and $x_{i}^{U, D}$ (which sets the darkfermion mass scale with respect to the messenger one).

Furthermore, it will be convenient to define a universal-flavor (UF) scenario, where one has up-down flavor universality in the mass sector of the colored messenger fields (i.e., $\bar{m}_{U}^{2}=\bar{m}_{D}^{2} \equiv$ $\bar{m}^{2}$ ). The latter is the most symmetric and predictive framework that one can envisage in the present model. We also define a nonuniversal flavor (NUF) scenario, where one relaxes the up and down flavor universality in the messenger sector, and assumes a universal $\bar{m}_{D}^{2}$ mass in the down sector which is independent from the universal $\bar{m}_{U}^{2}$ mass in the up sector.

\section{The $t \rightarrow(c, u) \bar{\gamma}$ decays}

In this section we analyze the FCNC decay of the top quark

$$
t \rightarrow q \bar{\gamma}
$$

where in the final state there can be either a $c$ or a $u$ quark. Using Eq. (40), the corresponding $\mathrm{BR}$, in the massless final-quark limit, can be parametrized in terms of the tree-level $\mathrm{BR}(t \rightarrow$ $W b)$, as follows

$$
\mathrm{BR}(t \rightarrow q \bar{\gamma})=\frac{\mathrm{BR}(t \rightarrow W b)}{\sqrt{2} G_{F}\left|V_{t b}\right|^{2} \rho\left(x_{W}\right)}\left(\frac{1}{\left(\Lambda_{L}^{t q}\right)^{2}}+\frac{1}{\left(\Lambda_{R}^{t q}\right)^{2}}\right)
$$

where $\rho(x)=(1-x)^{2}(1+2 x), \Lambda_{L, R}^{t u} \equiv\left(\Lambda_{L, R}^{U}\right)_{31}, \Lambda_{L, R}^{t c} \equiv\left(\Lambda_{L, R}^{U}\right)_{32}, x_{W}=\frac{M_{W}^{2}}{m_{t}^{2}}$, being $M_{W}$ and $m_{t}$ the $W^{ \pm}$and top-quark mass, respectively. The relevant $\Lambda_{L, R}^{t u}$ and $\Lambda_{L, R}^{t c}$ expressions are in Eq. (29).

Assuming a universal average messenger mass $\bar{m}_{U}=\bar{m}_{D}=\bar{m}$, the mass-scale dependence of $\mathrm{BR}(t \rightarrow q \bar{\gamma})$ turns out to be

$$
\operatorname{BR}(t \rightarrow q \bar{\gamma}) \propto \frac{m_{t}^{2}}{\bar{m}^{4} G_{F}}
$$

The lower allowed value of the average messenger mass $\bar{m}$ is constrained by dark-matter (DM) and vacuum-stability bounds, and, as a consequence, the $1 / \bar{m}^{4}$ term in Eq. (43) strongly suppresses the $t \rightarrow q \bar{\gamma}$ decay. In particular, we will prevent stable colored and EW messenger particles in the spectrum, which would conflict with DM constraints, hence allowing messenger decays into dark fermions according to the interaction Lagrangian in Eq. (19). In the following, by DM constraints we indicate the requirements that the mass spectrum is such that all the messenger decays are kinematically allowed.

\subsection{DM and vacuum stability constraints for $t \rightarrow q \bar{\gamma}$}

We now discuss the relevant theoretical bounds in the scalar messenger sector, and, in the following subsection, the corresponding upper bounds on $\mathrm{BR}(t \rightarrow q \bar{\gamma})$. We will assume, for the moment, up-down flavor universality (i.e., the UF scenario defined above). By using Eqs.(9) 
and (17) for the radiatively generated Yukawa couplings, we obtain the following prediction for the generic mass $M_{Q_{i}}$ of the dark fermion associated to the SM quark $q_{i}$, as a function of the quark mass $m_{i}$,

$$
M_{Q_{i}}=m_{i}\left(\frac{16 \pi^{2}}{g_{L} g_{R}}\right) \frac{1}{\xi f_{1}\left(x_{i}, \xi\right)},
$$

where $x_{i}=M_{Q_{i}}^{2} / \bar{m}^{2}$ and $\xi$ is the universal mixing parameter in the colored messenger sector. Note that, the quark masses $m_{i}$ as well as the running coupling constants $g_{L}, g_{R}$, appearing in Eqs. 44), 47), 49), and (50), are evaluated at the messenger mass scale $\mu \sim \bar{m}$.

Being $m_{ \pm}^{2}=\bar{m}^{2}(1 \pm \xi)$ the eigenvalues of the up-down degenerate messenger mass spectrum, in order to avoid stable messengers, the lightest messenger mass $m_{-}$must be larger than the mass of the heaviest dark fermion, that is $M_{Q_{t}}$, associated to the top-quark [4],

$$
m_{-} \geq M_{Q_{t}}
$$

On the other hand, the vacuum stability condition requires $\xi \leq 1$, in order to avoid either tachyons in the spectrum or color/charge-breaking minima through the generation of nonvanishing VEV in the messenger scalar sector [4]. Because of the $U(1)_{F}$ gauge invariance in the dark sector, Eq. (45) is sufficient to avoid stability for all messenger fields, and to guarantee that all dark fermions are stable particles. By using Eqs. (16) and (44), Eq. (45) can be rephrased into the following lower bound on the average messenger mass in the colored messenger sector

$$
\bar{m}^{2} \geq\left(\frac{16 \pi^{2}}{g_{L} g_{R}}\right)^{2} \frac{m_{t}^{2}}{\xi^{2} f_{1}^{2}\left(x_{t}, \xi\right)(1-\xi)},
$$

where $m_{t}$ is the top-quark mass. Notice that also the rhs of Eq. (46) depends on $\bar{m}$ through the ratio $x_{t}=M_{Q_{t}}^{2} / \bar{m}^{2}$ entering the loop function $f_{1}(x, \xi)$ defined in Eq. 18). At fixed $\xi$, the lowest $\bar{m}$ bound corresponds to equality in Eq. (45) and can be obtained by replacing $x_{t} \rightarrow 1-\xi$ inside $f_{1}\left(x_{t}, \xi\right)$ in Eq.46). The lowest $\bar{m}$ minimum in Eq. (46) is then a pure function of $\xi$, namely

$$
\bar{m} \geq m_{t}\left(\frac{16 \pi^{2}}{g_{L} g_{R}}\right) F(\xi),
$$

where $F(x)$ is given by

$$
F(x)=\frac{8 x \sqrt{1-x}}{2 x+(1-x)^{2} \log \left(\frac{1-x}{1+x}\right)} .
$$

For $x \ll 1$, the formula above simplifies to $F(x) \simeq 2 / x+1 / 3+\mathcal{O}(x)$, while, for $x \simeq 1$, one obtains $F(x) \simeq 4 \sqrt{1-x}+\mathcal{O}\left((1-x)^{3 / 2}\right)$.

By relaxing the full flavor universality in the messenger sector, and restricting mass degeneracy to the up and down messenger sectors separately, the above bounds in Eq.47) can be generalized as follows:

$$
\bar{m}_{U} \geq m_{t}\left(\frac{16 \pi^{2}}{g_{L} g_{R}}\right) F\left(\xi_{U}\right),
$$




$$
\bar{m}_{D} \geq m_{b}\left(\frac{16 \pi^{2}}{g_{L} g_{R}}\right) F\left(\xi_{D}\right),
$$

where $\bar{m}_{U(D)}$ and $\xi_{U(D)}$ refer to the up (down) sector. Notice that in the rhs of Eq. (50) the bottom-quark mass $m_{b}$ replaces $m_{t}$, since we are now assuming different average messenger masses (i.e., $\bar{m}_{U}^{2} \neq \bar{m}_{D}^{2}$ ) for the up and down sectors. A generalization of the above bounds to the leptonic sector is straightforward.

Accordingly, for $m_{t}=173.2 \mathrm{GeV}$ and a bottom-quark pole mass $m_{b}=4.78 \mathrm{GeV}$ [14], we find in the large $\xi_{U, D}$ regime

$$
\begin{aligned}
& \bar{m}_{U} \geq \frac{(110 \mathrm{TeV}) K_{t}(\bar{m})}{g_{L} g_{R}} \sqrt{1-\xi_{U}}, \\
& \bar{m}_{D} \geq \frac{(3 \mathrm{TeV}) K_{b}(\bar{m})}{g_{L} g_{R}} \sqrt{1-\xi_{D}}
\end{aligned}
$$

where the factors $K_{t, b}(\bar{m})<1$, defined by $m_{t, b}(\bar{m})=K_{t, b}(\bar{m}) m_{t, b}$, contain the renormalization effects connecting the top and bottom running masses $m_{t, b}(\bar{m})$ at the scale $\bar{m}$ with their pole masses $m_{t, b}$.

On the other hand, for $\xi \ll 1$ the lower bounds on $\bar{m}_{U, D}$ in Eqs. (49) and (50) are stronger, due to the enhancement factor $F(\xi) \sim 1 / \xi$ at small $\xi$. The singular behavior for $\xi \ll 1$ is a consequence of the vanishing of Yukawa couplings for $\xi \rightarrow 0$ at fixed dark fermion masses $M_{Q_{i}}$ (cf. Eq. (17)). Hence, large $M_{Q_{i}}$ values are needed to compensate the latter suppression in Eq. (17), and even larger $\bar{m}$ values due to the DM constraints in Eq. (45). Note that, if we assume flavor universality in the up and down sector for messenger fields, then the strongest bound on $\bar{m}$ in Eq. (49) applies. In the UF scenario, since the $t \rightarrow q \bar{\gamma}$ width scales as $1 / \bar{m}^{4}$ (cf. Eq. (43)), the corresponding $\mathrm{BR}(t \rightarrow q \bar{\gamma})$ will be severely constrained, especially for small $\xi$ mixing.

Since, for sufficiently large mixing, the limits in Eqs. (51)-(52) might go below the messenger mass bounds arising from their nonobservation in direct pair production at the LHC, we will distinguish in our study the $\xi$ ranges that correspond to lower mass limits that could be in conflict with the LHC results. Actually, although the present model shows features that are similar to the SUSY phenomenology, the actual LHC mass bounds depend nontrivially on the model parameters. Dedicated LHC analyzes will be needed in order to set robust bounds on the corresponding particle and parameter spectra. Then, in our analysis we will just assume a few tentative mass bounds the could be derived for messenger searches at the LHC and set the corresponding maximal $\xi$ mixing value not to overcome these tentative bounds. In particular, in the following we will assume that the LHC presently excludes pair production of colored messengers lighter than $1 \mathrm{TeV}$ and of colorless (EW) messengers lighter than $300 \mathrm{GeV}$.

In the numerical analysis of the following sections, since we aim at a simplified LO analysis, we will not include the QCD running of relevant couplings and masses. Hence, the numerical behavior reported in all tables and figures will correspond to setting all quark masses to their pole mass values. 


\begin{tabular}{|c||c|c|c|}
\hline$\xi$ & $\mathrm{BR}^{\max }(t \rightarrow q \bar{\gamma})$ & $\bar{m}^{\min }[\mathrm{TeV}]$ & $m_{-}^{\min }[\mathrm{TeV}]$ \\
\hline 0.1 & $5.6 \times 10^{-15}$ & 554 & 526 \\
\hline 0.2 & $1.0 \times 10^{-13}$ & 279 & 249 \\
\hline 0.3 & $6.0 \times 10^{-13}$ & 185 & 155 \\
\hline 0.5 & $7.5 \times 10^{-12}$ & 107 & 75 \\
\hline 0.7 & $7.0 \times 10^{-11}$ & 67 & 37 \\
\hline 0.8 & $2.5 \times 10^{-10}$ & 52 & 23 \\
\hline 0.9 & $1.6 \times 10^{-9}$ & 35 & 11 \\
\hline 0.95 & $8.3 \times 10^{-9}$ & 25 & 5.5 \\
\hline 0.99 & $2.6 \times 10^{-7}$ & 11 & 1.1 \\
\hline
\end{tabular}

Table 2: Maximum values of $\operatorname{BR}(t \rightarrow q \bar{\gamma})$ in the UF scenario allowed by vacuum stability and DM constraints, corresponding to the minimum allowed average messenger mass $\bar{m}^{\text {min }}$, and to the lightest up-down universal messenger mass eigenvalue $m_{-}^{\min }=\bar{m}^{\min } \sqrt{1-\xi}$ versus the mixing parameter $\xi$. Results are in unit of couplings, that is they assume $\bar{e} \bar{e}_{3}^{U}=g_{L, R}=\rho_{L, R}^{33,13,23}=1$, with all other elements of flavor matrices set to zero.

\subsection{Upper bounds on $\operatorname{BR}(t \rightarrow q \bar{\gamma})$}

A rough estimate of the upper bounds on $\operatorname{BR}(t \rightarrow q \bar{\gamma})$, versus the relevant free parameters of the model, needs a few working assumptions. In the UF scenario (that is for $\bar{m}_{U}^{2}=\bar{m}_{D}^{2} \equiv \bar{m}^{2}$ and $\left.\xi_{U}=\xi_{D} \equiv \xi\right)$, we can see that in the rhs of the two equations entering Eq. (29) for $\Lambda_{L, R}^{U}$ [or equivalently in Eq. (30) for $\Lambda_{L, R}^{D}$ ] the first terms in parenthesis are dominant over the second ones, being the latter suppressed by the loop factor $g_{R / L}^{2} / 16 \pi^{2}$. Since $F_{L R / R L}$ and $F_{L L / R R}$ are almost of the same order, we can safely neglect the contribution of the second terms in Eq. (29). In order to further simplify the analysis, one can also assume universality between the $L / R$ quark couplings to dark fermions (i.e., $g_{L}=g_{R}$ ), and the $\rho_{L, R}$ matrix elements (i.e., $\left.\left(\rho_{L}\right)_{j i}=\left(\rho_{R}\right)_{j i}\right)$.

Under the UF assumption and neglecting $g_{R / L}^{2} / 16 \pi^{2}$ terms, disregarding overall factors from couplings, the generic $q \rightarrow q^{\prime} \bar{\gamma}$ width depends on three fundamental parameters, i.e., the average messenger mass $\bar{m}$, the mixing parameters $\xi_{U}$, and $x_{3}^{U}$, satisfying the conditions in Eqs. (47). Then, since $\operatorname{BR}(t \rightarrow q \bar{\gamma}) \sim 1 / \bar{m}^{4}$, the largest allowed BR upper bound corresponds to the equality condition in Eq. (51). Analogous conclusions hold for the FCNC decay in dark photon in the down-quark sector.

In Table 2, we report the results for the maximum allowed $\operatorname{BR}(t \rightarrow q \bar{\gamma})$, satisfying the vacuum stability bounds and DM constraints, versus the mixing parameter $\xi=\xi_{U}$. The results assume $U(1)_{F}$ charges and other multiplicative couplings normalized to 1 . In particular, in Table 2, we set $g_{L, R}=1$, and $\bar{e} \bar{e}_{3}^{U}=\rho_{L, R}^{33,13,23}=1$, with all other elements of flavor matrices set to zero. In the last two columns we report $\bar{m}^{\text {min }}$, the minimum $\bar{m}$ allowed by DM constraints, and the minimum $m_{-}^{\text {min }}$ of the corresponding lowest messenger mass eigenvalue, as defined in Eq. (16).

The resulting allowed $\operatorname{BR}(t \rightarrow q \bar{\gamma})$ values get tiny for small mixing $\xi_{U}$, but might approach detectability at future colliders in case one assumes a quite large mixing (which is typical of 


\begin{tabular}{|c||c|c|c|}
\hline$\xi_{D}$ & $\mathrm{BR}^{\max }(t \rightarrow q \bar{\gamma})$ & $\bar{m}_{D}^{\min }[\mathrm{TeV}]$ & $m_{D_{-}}^{\min }[\mathrm{TeV}]$ \\
\hline 0.1 & $1.2 \times 10^{-14}$ & 15 & 14 \\
\hline 0.2 & $2.1 \times 10^{-13}$ & 7.7 & 6.9 \\
\hline 0.3 & $1.3 \times 10^{-12}$ & 5.1 & 4.3 \\
\hline 0.5 & $1.8 \times 10^{-11}$ & 2.9 & 2.1 \\
\hline 0.7 & $2.4 \times 10^{-10}$ & 1.9 & 1.0 \\
\hline$(0.8)$ & $1.3 \times 10^{-9}$ & 1.4 & 0.64 \\
\hline$(0.9)$ & $2.0 \times 10^{-8}$ & 0.97 & 0.31 \\
\hline$(0.95)$ & $3.1 \times 10^{-7}$ & 0.68 & 0.15 \\
\hline$(0.99)$ & $1.8 \times 10^{-4}$ & 0.30 & 0.03 \\
\hline
\end{tabular}

Table 3: Maximum values of $\operatorname{BR}(t \rightarrow q \bar{\gamma})$ in the NUF scenario allowed by vacuum stability and DM constraints, corresponding to the minimum allowed average messenger mass $\bar{m}_{D}^{\min }$, and to the lightest down messenger mass eigenvalue $m_{D_{-}}^{\min }=\bar{m}_{D}^{\min } \sqrt{1-\xi_{D}}$, versus the mixing parameter $\xi_{D}$. Results are in unit of couplings, that is they assume $\bar{e} \bar{e}_{3}^{D}=g_{L, R}=\eta_{L, R}^{33,13,23}=1$, with all other elements of flavor matrices set to zero. Values of $\xi_{D}$ in parenthesis might be excluded by direct searches of colored scalar particles.

natural theories [1] $)$. Indeed, for $\xi_{U}=0.95$ one can achieve a BR, in unit of couplings, of the order of $10^{-8}$, which can go up to values $\sim 10^{-7}$ for $\xi_{U}=0.99$. These bounds are effective for couplings of the order $\mathcal{O}(1)$, and in the more realistic case of perturbative smaller couplings they could be even more severe. On the other hand, there are theoretical arguments suggesting the values $\bar{\alpha} \sim(0.05-0.2)$, while large $\xi$ mixings and $g_{L, R} \sim \mathcal{O}(1)$ are favored in order to avoid large corrections to the Higgs-boson mass [4, 12]. Therefore, the effect of a more realistic couplingconstant normalization in the present scenario can induce a suppression of order $\left(10^{-1}-10^{-2}\right)$ on the BR upper bounds in Table 2, modulo possible small values of $\rho_{L, R}^{13,23}$.

We now relax the up and down flavor universality in the messenger sector, and assume a universal $\bar{m}_{D}^{2}$ mass in the down sector independent from the universal $\bar{m}_{U}^{2}$ mass in the up sector (the NUF scenario defined above). Then, the DM constraints on $\bar{m}_{D}$ are less severe according to Eq. (50), and allow lighter messenger masses in the down sector, which would in turn permit a larger $\operatorname{BR}(t \rightarrow q \bar{\gamma})$. Indeed, $\bar{m}_{D}$ enters the $\Lambda_{R}$ scale in Eq.29) which receives contributions from both the down and up messenger sectors.

In Table 3 we show the maximum $\operatorname{BR}(t \rightarrow q \bar{\gamma})$ allowed in the NUF scenario, versus $\xi_{D}$, computed using $\bar{m}_{D}$ given by the equality in Eq.(50). We have neglected the contributions induced by the $1 / \Lambda_{L}^{t q}$ scale (which are suppressed by terms $\sim 1 / \bar{m}_{U}^{2}$ ), and retained only the $F_{L L}$ contribution in $1 / \Lambda_{R}^{t q}$. We remind that the $F_{L L}$ term comes from the chirality flip contribution to the FC magnetic-dipole operator induced by the external states, and thus is suppressed with respect to other contributions by a loop factor $\sim g_{L}^{2} /\left(16 \pi^{2}\right)$. Despite the suppression factor $1 /\left(16 \pi^{2}\right)$, the upper bounds on the $\mathrm{BR}(t \rightarrow q \bar{\gamma})$ in Table 3 are more relaxed than the UFscenario ones in Table 2, since $\bar{m}_{D}$ can be much lower than $\bar{m}_{U}$ in the NUF scenario. Values $\xi_{D}>0.7$ (shown in parenthesis in Table 3 ) might be excluded by direct searches of colored scalar particles at the LHC, since they correspond to light messenger masses in the down sector below 

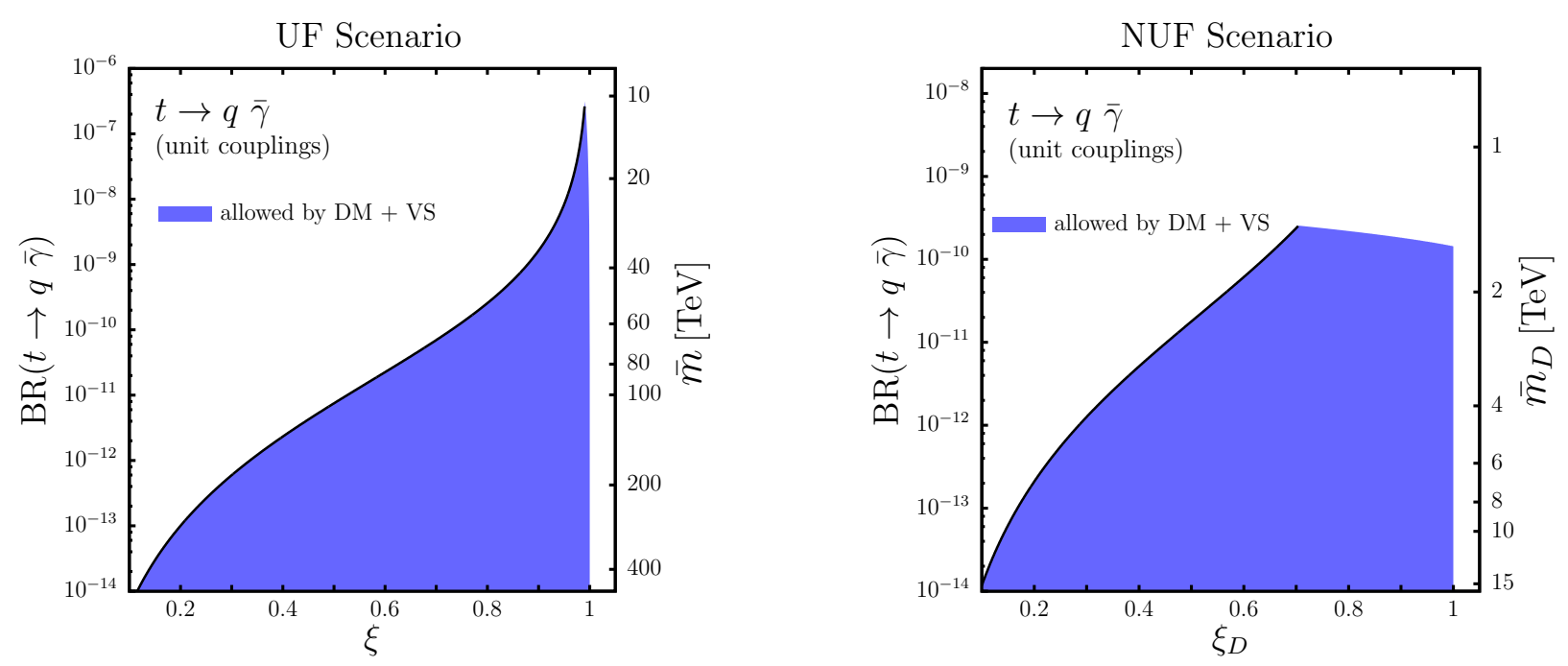

Figure 3: Allowed regions (colored areas) by DM and vacuum stability (VS) constraints for $\mathrm{BR}(t \rightarrow q \bar{\gamma})$ and for the average messenger mass scales $\bar{m}$ and $\bar{m}_{D}$ versus the corresponding mixing $\xi$ and $\xi_{D}$, in the UF (left) and NUF (right) scenarios, respectively.

$1 \mathrm{TeV}$. Anyway, a dedicated search able to substantiate the latter statement (which depends on model-dependent features) has not yet been performed at the LHC.

We summarize the above results in Fig. 3, where we show the regions of $\operatorname{BR}(t \rightarrow q \bar{\gamma})$ and relevant average messenger mass $\left(\bar{m}\right.$ and $\bar{m}_{D}$ for the UF and NUF scenarios respectively) allowed by the DM and vacuum stability constraints versus the mixing parameters $\xi$ and $\xi_{D}$, in the UF and NUF scenarios, respectively. Notice that, at fixed mixing, the black bold upper line in the blue region gives, on the left vertical axis, the upper bound on $\operatorname{BR}(t \rightarrow q \bar{\gamma})$, and, on the right vertical axis, the corresponding lower $\bar{m}_{D}$ value. The upper bound for $\xi_{D}>0.7$ in the left plot is ruled by direct searches of colored scalar particles at the LHC, since it corresponds to light messenger masses in the down sector of $1 \mathrm{TeV}$.

We now discuss the constraints coming from possible dark-fermion and messenger contributions to the FCNC decays $t \rightarrow q \gamma$, where the dark photon is replaced by a SM photon in the final state. In the SM this channel receives the main contribution from $W$ and $b$-quark loops, whose amplitude, due to the Glashow-Iliopoulos-Maiani (GIM) mechanism [16], is suppressed by terms $\sim V_{t s} m_{b}^{2} / M_{W}^{2}$ (where $V_{t s}$ is the CKM matrix element), which makes the corresponding decay rate quite small.

The $\mathrm{SM}$ values of $\mathrm{BR}(t \rightarrow c \gamma)$ and $\mathrm{BR}(t \rightarrow u \gamma)$ are a few $10^{-14}$ and a few $10^{-16}$, respectively [17]. However, in the present framework, $t \rightarrow q \gamma$ would receive extra contributions from loops of messengers and dark fermions, involving the same flavor structures entering the $t \rightarrow q \bar{\gamma}$ amplitude (see Appendix for details). We will then assume that these further contributions to the $t \rightarrow q \gamma$ amplitude are dominant with respect to the SM one, and apply the present experimental constraints on $\mathrm{BR}(t \rightarrow q \gamma)$ to indirectly constrain the $t \rightarrow q \bar{\gamma}$ decay rate.

Analytical results for the extra $t \rightarrow q \gamma$ amplitude are reported in the Appendix, by retaining only the dominant contributions proportional to the dark-fermion masses. By applying the same 
approximation to the $t \rightarrow q \bar{\gamma}$ amplitude, we get a simplified relation that connects the two BR's by the following expression:

$$
\mathrm{BR}(t \rightarrow q \bar{\gamma})=\frac{\bar{\alpha}}{\alpha}\left(\frac{\bar{e}_{3}^{U} f_{2}\left(x_{3}^{U}, \xi_{U}\right)}{e_{U} \bar{f}_{2}\left(x_{3}^{U}, \xi_{U}\right)}\right)^{2} \mathrm{BR}(t \rightarrow q \gamma),
$$

where $\alpha=1 / 137$ is the electromagnetic (EM) fine structure constant, $e_{U}=2 / 3$ is the top-quark EM charge, $f_{2}(x, y)$ is given in Eq. (33), and $\bar{f}_{2}(x, y)$ is derived in the Appendix. Notice that, in Eq. (53), the factor connecting the two BR's does not depend on the flavor matrices, since the latter are the same for the dominant contributions to the two processes, and approximately cancel out in the $\mathrm{BR}$ ratio. Then, neglecting the $\mathrm{SM}$ contributions, theoretical $\mathrm{BR}(t \rightarrow q \gamma)$ upper bounds versus the relevant model parameters can be obtained from Tables 2 and 3 , by means of Eq. (53).

Conversely, the LHC present constraints on $\mathrm{BR}(t \rightarrow q \gamma)$ can set indirect experimental upper bounds on $\operatorname{BR}(t \rightarrow q \bar{\gamma})$ versus $x_{3}^{U}$ and $\xi_{U}$, by means of Eq.(53). The present $\operatorname{BR}(t \rightarrow q \gamma)$ upper limits at $95 \%$ C.L., reported by the CMS collaboration, are [18]

$$
\begin{aligned}
& \mathrm{BR}^{\exp }(t \rightarrow u \gamma)<1.3 \times 10^{-4} \\
& \mathrm{BR}^{\exp }(t \rightarrow c \gamma)<1.7 \times 10^{-3}
\end{aligned}
$$

Actually, the stringent DM constraints in Eqs. (49)-(50) set quite strong upper limits on $\mathrm{BR}(t \rightarrow q \bar{\gamma})$, and push a possible NP contribution to $t \rightarrow q \gamma$ in this scenario well below the present experimental sensitivity to this channel. On the contrary, if we relax DM constraints, and assume that NP contributions completely saturate the $\mathrm{BR}^{\exp }(t \rightarrow q \gamma)$ experimental limits in Eq. (55), we can derive indirect experimental $\mathrm{BR}(t \rightarrow q \bar{\gamma})$ upper bounds versus $\bar{\alpha}, \xi_{U}$, and $x_{3}^{U}$. For instance, in the UF scenario, assuming $\left(\bar{e}_{3}^{U}\right)^{2} \bar{\alpha} \sim 0.1$ as a reference value for the relevant combination of $U(1)_{F}$ couplings, as indicated by naturalness arguments (see Appendix in [12]), we get the following upper bounds on $\operatorname{BR}(t \rightarrow q \bar{\gamma})$, for representative $\xi_{U}$ and $x_{3}^{U}$ values:

- for $\xi_{U}=0.1$, and $x_{3}^{U}=0.8$ (small-mixing regime)

$$
\begin{aligned}
\mathrm{BR}^{(t \rightarrow u \gamma)}(t \rightarrow u \bar{\gamma}) & <1.8 \times 10^{-2}\left(\frac{\bar{\alpha}}{0.1}\right) \\
\mathrm{BR}^{(t \rightarrow c \gamma)}(t \rightarrow c \bar{\gamma}) & <2.3 \times 10^{-1}\left(\frac{\bar{\alpha}}{0.1}\right)
\end{aligned}
$$

- for $\xi_{U}=0.8$, and $x_{3}^{U}=0.1$ (large-mixing regime)

$$
\begin{aligned}
\mathrm{BR}^{(t \rightarrow u \gamma)}(t \rightarrow u \bar{\gamma}) & <3.4 \times 10^{-2}\left(\frac{\bar{\alpha}}{0.1}\right) \\
\mathrm{BR}^{(t \rightarrow c \gamma)}(t \rightarrow c \bar{\gamma}) & <4.4 \times 10^{-1}\left(\frac{\bar{\alpha}}{0.1}\right) .
\end{aligned}
$$

The resulting upper bounds are much weaker than the ones in Tables 2 and 3 set by DM 
constraints 4 . Note that such large values of the upper bounds overwhelm the possibility of having extra top decay channels allowed by the present measurement of $\mathrm{BR}(t \rightarrow W b)$ [14].

In conclusion, by imposing vacuum stability and DM constraints, we expect that allowed $\mathrm{BR}(t \rightarrow q \bar{\gamma})$ values do not exceed $\sim\left(10^{-8}-10^{-7}\right)$, which are barely close to the HL-LHC maximum experimental sensitivity on rare top-quark processes, but might be well inside the exploration domain of a future hadron collider at $100 \mathrm{TeV}$ [19]. However, larger $\operatorname{BR}(t \rightarrow q \bar{\gamma})$ values, up to $\left(10^{-5}-10^{-4}\right)$, could in principle be achieved, provided the LHC constraints on colored scalar particle production can be avoided in case of messengers that are lighter than 1 $\mathrm{TeV}$ ( $c f$. Table 3). On the other hand, in case one can evade both DM constraints and LHC direct bounds on colored scalar production, the expected $\mathrm{BR}(t \rightarrow q \bar{\gamma})$ range is essentially just limited by the present accuracy on the measurement of $\mathrm{BR}(t \rightarrow W b)$.

\section{$5 \quad$ The $b \rightarrow(s, d) \bar{\gamma}$ decays}

Here we analyze the FCNC decay $b \rightarrow q \bar{\gamma}$, with $q=s, d$. Its total width is given by Eq.(39), with $i=3$ and $j=2,1$ for the $q=s, d$ transitions, respectively. The corresponding BR can conventionally be expressed in terms of $\mathrm{BR}^{\exp }\left(B \rightarrow X_{c} \bar{\nu} e\right)=(10.65 \pm 0.16) \%$, the world-average measurement of the $B$-meson semileptonic BR [14]-[15]. To this aim, the tree-level semileptonic $b \rightarrow c e \bar{\nu}$ decay width, $\Gamma_{0}^{b}$, can be expressed through

$$
\Gamma_{0}^{b}=\frac{G_{F}^{2} m_{b}^{5}\left|V_{c b}\right|^{2}}{192 \pi^{3}} f_{1}\left(z_{c b}\right)
$$

where $f_{1}(x)=1-8 x+8 x^{3}-x^{4}-12 x^{2} \log x$, with $z_{c b}=m_{c}^{2} / m_{b}^{2}$, and $V_{c b}$ is the relevant CKM matrix element. Then, one has

$$
\operatorname{BR}(b \rightarrow q \bar{\gamma})=\frac{12 \mathrm{BR}^{\exp }\left(B \rightarrow X_{c} \bar{\nu} e\right)}{G_{F}^{2}\left|V_{c b}\right|^{2} m_{b}^{2} f_{1}\left(z_{c b}\right)}\left(\frac{1}{\left(\Lambda_{L}^{b q}\right)^{2}}+\frac{1}{\left(\Lambda_{R}^{b q}\right)^{2}}\right)
$$

with $q=s, d$. The expressions needed for $\Lambda_{L, R}^{b s} \equiv\left(\Lambda_{L, R}^{D}\right)_{32}$, and $\Lambda_{L, R}^{b d} \equiv\left(\Lambda_{L, R}^{D}\right)_{31}$ can be found in Eq. 30$)$. Note that the $\operatorname{BR}(b \rightarrow q \bar{\gamma})$ dominant $m_{b}$ dependence cancels out in $1 /\left(\Lambda_{L, R}^{b q}\right)^{2}$, since the Yukawa couplings are generated radiatively. For our numerical analysis, we use the central values of the $c$-quark and $b$-quark pole masses, $m_{c}=1.67 \mathrm{GeV}$ and $m_{b}=4.78 \mathrm{GeV}$, respectively, and the $V_{c b}$ central value $V_{c b}=(42.46 \pm 0.88) \times 10^{-3}$, extracted from the $B$ semileptonic BR reported above [14]-[15].

\footnotetext{
${ }^{4}$ Notice that the $\mathrm{BR}^{(t \rightarrow q \gamma)}(t \rightarrow q \bar{\gamma})$ upper bounds derived from the present experimental $\operatorname{BR}(t \rightarrow q \gamma)$ constraints increases by decreasing the $x_{3}^{U}$, thanks to the $\log x_{3}^{U}$ enhancement of $\operatorname{BR}(t \rightarrow q \bar{\gamma})$ with respect to $\mathrm{BR}(t \rightarrow q \gamma)$. In the $t \rightarrow q \bar{\gamma}$ amplitude, the $\log x_{3}^{U}$ term in the loop function $f_{2}\left(x_{3}^{U}, \xi_{U}\right)$ defined in Eq. (33) is due to an infrared effect in the diagrams where a dark photon is radiated from internal dark-fermion lines. Indeed, at small $x$, the $f_{2}(x, \xi)$ function behaves as $f_{2}(x, \xi) \simeq \frac{2 \xi}{1-\xi}(1+\log x-\log (1-\xi))+\mathcal{O}(x)$. The $\log x$ term is absent in the corresponding $t \rightarrow q \gamma$ loop function $\bar{f}_{2}(x, \xi)$ Eq. 119$)$, since dark fermions are not charged under EM interactions.
} 


\subsection{DM and vacuum stability constraints for $b \rightarrow q \bar{\gamma}$}

Following the same approach adopted for the top-quark decays described in the previous section, we now present the theoretical $\operatorname{BR}(b \rightarrow q \bar{\gamma})$ upper bounds. We neglect the second term in the square brackets in Eq. (30), which is of order $\sim g_{L, R}^{2} /\left(16 \pi^{2}\right)$. Contrary to the top-quark case, we can neglect the latter term in the NUF scenario as well, since no enhancement is expected in the corresponding contributions in the $b$-quark case, not even in the one proportional to the $\rho_{L}$ matrix elements in Eq. (30) (the latter being suppressed by $1 / \bar{m}_{U}^{2}$, which is typically smaller than $\left.1 / \bar{m}_{D}^{2}\right)$.

In order to simplify the analysis, we set all couplings (including the flavor matrix elements $\left.\rho_{L, R}\right)$ to 1 , and consider only the dependence on the average messenger mass and corresponding mixing parameter in the down messenger sector. We consider first the UF scenario in which $\bar{m}_{D}^{2}=\bar{m}_{U}^{2}$, and $\xi_{D}=\xi_{U}$. We also assume symmetric left-right couplings, $g_{L}=g_{R}$, and left-right flavor matrices, $\rho_{L}=\rho_{R}$. Then, $\operatorname{BR}(b \rightarrow q \bar{\gamma})$ gets its maximum for the minimum allowed $\bar{m}_{D}$. According to Eq. (51), for large $\xi_{U}$, this corresponds to $\bar{m}_{D}^{\min }=110 \mathrm{TeV} \sqrt{1-\xi_{D}}$ for $g_{L, R}=1$, and $K_{b}(\bar{m})=1$.

Notice that the relevant dark-fermion mass entering the FCNC $b$ decays is the heaviest dark fermion associated to the down sector, which appears through the $x_{3}^{D}$ dependence of the loop functions. In the UF hypothesis, we can relate the $x_{3}^{U}$ and $x_{3}^{D}$ variables by assuming that the dark fermion masses are approximately a rescaled version of the SM fermion masses. This is a realistic approximation since the $C_{0}(x)$ loop function in Eq. (11) has a weak $x$ dependence in the range $0<x<1$. Then, for assessing $\operatorname{BR}(b \rightarrow q \bar{\gamma})$ upper bounds in the UF scenario, we will assume the following approximated relation:

$$
x_{3}^{D} \simeq x_{3}^{U} \frac{m_{b}^{2}}{m_{t}^{2}} .
$$

In Table 4 , the $\mathrm{BR}(b \rightarrow q \bar{\gamma})$ upper bounds induced by vacuum stability and DM constraints in the UF scenario are presented, as a function of the mixing parameter $\xi_{D}$. These results hold for unit couplings. For arbitrary couplings, the results in Table 4 must be multiplied by the product $\left(\bar{e} \bar{e}_{3}^{D} \eta_{L}^{j 3} / \eta_{L}^{33}\right)^{2}$, or analogously $\left(\bar{e} \bar{e}_{3}^{D} \eta_{R}^{j 3} / \eta_{R}^{33}\right)^{2}$, with $j=1,2$.

On the other hand, in the NUF scenario, lower $\bar{m}_{D}$ values are allowed by vacuum stability and DM constraints, and quite larger $\operatorname{BR}(b \rightarrow q \bar{\gamma})$ values can be reached. The NUF scenario results versus $\xi_{D}$ are presented in Table 5. One can see that particularly large $\operatorname{BR}(b \rightarrow q \bar{\gamma})$ values are allowed in case of large $\xi_{D}$ mixing, that are possibly well inside the discovery range of future $\mathrm{B}$ factories and FCC-ee. On the other hand, an experimental bound $\mathrm{BR}\left(b \rightarrow s \mathrm{X}_{\text {inv }}\right)<$ $\mathcal{O}(10 \%)$ [20] (where $\mathrm{X}_{\mathrm{inv}}$ stands for the inclusive invisible channe ${ }^{5}$ ) might exclude the range $\xi_{D} \gtrsim 0.6$, when all relevant couplings are set to 1 .

\section{2 $\operatorname{BR}(b \rightarrow s \bar{\gamma})$ upper bounds from the $\mathbf{B R}(b \rightarrow s \gamma)$ measurement}

We now consider the experimental constraints coming from the measurement of the $b \rightarrow s \gamma$ decay rate into a photon, and in particular the bounds on $\mathrm{NP}$ contributions to $\mathrm{BR}(b \rightarrow s \gamma)$.

\footnotetext{
${ }^{5}$ In present experimental analysis, kinematical distributions are according to SM, where $X_{\text {inv }}$ is given by $\nu \bar{\nu}$ pairs. For the possibility to constrain nonstandard final states with $X_{\text {inv }}$, see [21].
} 


\begin{tabular}{|c||c|c|c|}
\hline$\xi$ & $\mathrm{BR}^{\max }(b \rightarrow q \bar{\gamma})$ & $\bar{m}^{\min }[\mathrm{TeV}]$ & $m_{-}^{\min }[\mathrm{TeV}]$ \\
\hline 0.1 & $7.5 \times 10^{-9}$ & 554 & 526 \\
\hline 0.2 & $1.2 \times 10^{-7}$ & 279 & 249 \\
\hline 0.3 & $6.5 \times 10^{-7}$ & 185 & 155 \\
\hline 0.5 & $6.2 \times 10^{-6}$ & 107 & 75 \\
\hline 0.8 & $1.2 \times 10^{-4}$ & 52 & 23 \\
\hline 0.9 & $6.0 \times 10^{-4}$ & 35 & 11 \\
\hline 0.95 & $2.6 \times 10^{-3}$ & 25 & 5.5 \\
\hline 0.99 & $6.7 \times 10^{-2}$ & 11 & 1.1 \\
\hline
\end{tabular}

Table 4: Maximum allowed $\operatorname{BR}(b \rightarrow q \bar{\gamma})$ after applying vacuum stability and DM constraints, corresponding to the minimum allowed average mass $\bar{m}^{\text {min }}$, and to the lightest universal messenger mass eigenvalue $m_{-}^{\min }=\bar{m}^{\min } \sqrt{1-\xi}$, versus the mixing parameter $\xi$, in the UF scenario. Results are in unit of couplings, that is they assume $\bar{e} \bar{e}_{3}^{U}=g_{L, R}=\eta_{L, R}^{33,13,23}=1$, with all other flavor matrix elements set to zero.

\begin{tabular}{|c||c|c|c|}
\hline$\xi_{D}$ & $\mathrm{BR}^{\max }(b \rightarrow q \bar{\gamma})$ & $\bar{m}_{D}^{\min }[\mathrm{TeV}]$ & $m_{D_{-}}^{\min }[\mathrm{TeV}]$ \\
\hline 0.1 & $5.8 \times 10^{-5}$ & 15 & 14 \\
\hline 0.2 & $1.1 \times 10^{-3}$ & 7.7 & 6.9 \\
\hline 0.3 & $6.3 \times 10^{-3}$ & 5.1 & 4.3 \\
\hline 0.5 & $7.9 \times 10^{-2}$ & 2.9 & 2.1 \\
\hline$(0.6)$ & $2.4 \times 10^{-1}$ & 2.3 & 1.5 \\
\hline
\end{tabular}

Table 5: Results as in Table 4 but for the NUF scenario, where we assume $\bar{e} \bar{e}_{3}^{D}=g_{L, R}=$ $\eta_{L, R}^{33,13,23}=1$, with all other flavor matrix elements set to zero. The range $\xi_{D} \geq 0.6$ might be excluded by the condition $\mathrm{BR}\left(b \rightarrow s \mathrm{X}_{\mathrm{inv}}\right)<\mathcal{O}(10 \%)$, where $\mathrm{X}_{\mathrm{inv}}$ stands for inclusive invisible particles (see text). 
The $b \rightarrow s \gamma$ process is known with high precision in the SM, with a next-to-next-to-leadingorder (NNLO) accuracy in QCD (see e.g. [22] for a complete review on the subject). The most updated SM theoretical prediction provides the value [23]

$$
\operatorname{BR}\left(B \rightarrow X_{S} \gamma\right)=(3.36 \pm 0.23) \times 10^{-4}
$$

The effective low-energy Hamiltonian for the $\Delta B=1$ transitions, describing the $b \rightarrow s \gamma$ decay, is given by

$$
H_{e f f}^{\Delta B=1}=-\frac{4 G_{F}}{\sqrt{2}} V_{32}^{\star} V_{33}^{\star} \sum_{i=1}^{8} C_{i}\left(\mu_{b}\right) Q_{i}\left(\mu_{b}\right),
$$

where the complete basis of operators $Q_{i}$ in the SM can be found e.g. in [24]. The Wilson coefficients $C_{i}\left(\mu_{b}\right)$ are evaluated at the low-energy scale $\mu_{b} \sim \mathcal{O}\left(m_{b}\right)$ and have been computed at the NNLO in QCD [22]. The $Q_{7}$ and $Q_{8}$ operators (conventionally, the magnetic-dipole and chromagnetic-dipole operators, respectively) are the main operators receiving contributions from NP, as occurs in our scenario, and are defined as

$$
\begin{aligned}
Q_{7} & =\frac{e}{16 \pi^{2}} m_{b}\left(\bar{s}_{L} \sigma^{\mu \nu} b_{R}\right) F_{\mu \nu} \\
Q_{8} & =\frac{g_{S}}{16 \pi^{2}} m_{b}\left(\bar{s}_{L} \sigma^{\mu \nu} T^{a} b_{R}\right) G_{\mu \nu}^{a}
\end{aligned}
$$

where $F_{\mu \nu}, G_{\mu \nu}^{a}$ are the EM and QCD field strengths, respectively, with $a=1, \ldots, 8$ running on the adjoint representation of the QCD $S U(3)_{c}$ group.

The present NP scenario will give a contribution at one loop to the Wilson coefficients of the $Q_{7}$ and $Q_{8}$ operators at the $M_{W}$ scale, namely to $C_{7}\left(M_{W}\right)$ and $C_{8}\left(M_{W}\right)$, respectively. The corresponding $b \rightarrow s \gamma$ and $b \rightarrow s g$ decay amplitudes induced by these operators (with $g$ standing for a gluon) can be found in the Appendix. However, the present model induces also contributions to two new local operators $\tilde{Q}_{7}$ and $\tilde{Q}_{8}$, which are defined by assuming an opposite chirality structure in Eq. 65] [28]. We will refer to $\tilde{C}_{7}\left(M_{W}\right)$ and $\tilde{C}_{8}\left(M_{W}\right)$ as the Wilson coefficients corresponding to $Q_{7}$ and $\tilde{Q}_{8}$ at the $M_{W}$ scale.

NP effects in $b \rightarrow s \gamma$ can be parametrized in a model-independent way by introducing the $R_{7,8}$ and $\tilde{R}_{7,8}$ parameters defined at the EW scale as

$$
R_{7,8} \equiv \frac{C_{7,8}^{\mathrm{NP}}\left(M_{W}\right)}{C_{7,8}^{\mathrm{SM}}\left(M_{W}\right)}, \quad \tilde{R}_{7,8} \equiv \frac{\tilde{C}_{7,8}^{N P}\left(M_{W}\right)}{C_{7,8}^{\mathrm{SM}}\left(M_{W}\right)},
$$

where $C_{7,8}^{\mathrm{NP}}$ include the pure NP contribution. The Wilson coefficients above are meant to be evaluated at the leading order (LO). We are now considering their effect on $\operatorname{BR}\left(B \rightarrow X_{s} \gamma\right)$ evaluated at the next-to-leading order (NLO) [25], where nonperturbative $1 / m_{b}$ [27] and $1 / m_{c}$ [26] corrections have been included. Although the $b \rightarrow s \gamma$ rate is known at the NNLO [23], the LO accuracy for NP effects is sufficient for the purposes of the present analysis. Indeed, we restricted to a 1-loop matching, while a true NLO accuracy in the NP effects would require a (nontrivial to perform) 2-loop matching. 
By inserting the $R_{7,8}$ and $\tilde{R}_{7,8}$ definition in the final $\mathrm{BR}\left(B \rightarrow X_{s} \gamma\right)$ expression, as in [25], one obtains [28]

$$
\begin{aligned}
\mathrm{BR}\left(B \rightarrow X_{S} \gamma\right) & =(3.36 \pm 0.26) \times 10^{-4}\left(1+0.622 R_{7}+0.090\left(R_{7}^{2}+\tilde{R}_{7}^{2}\right)\right. \\
& \left.+0.066 R_{8}+0.019\left(R_{7} R_{8}+\tilde{R}_{7} \tilde{R}_{8}\right)+0.002\left(R_{8}^{2}+\tilde{R}_{8}^{2}\right)\right)
\end{aligned}
$$

where, with respect to [28], we rescaled the SM central value by the most updated result at the NNLO accuracy [23], and kept the $(1-\sigma)$ SM uncertainty.

The experimental measurements of the CP- and isospin-averaged $\mathrm{BR}\left(B \rightarrow X_{s} \gamma\right)$ by CLEO [29], Belle [30], and BABAR [31] lead to the combined value [32]

$$
\operatorname{BR}^{\exp }\left(B \rightarrow X_{S} \gamma\right)=(3.43 \pm 0.21 \pm 0.07) \times 10^{-4}
$$

In order to constrain the contributions induced by the present NP scenario, we will make a few simplifying assumptions. As can be seen from the coefficients multiplying the $R_{i}$ and $R_{i} R_{j}$ terms in the right-hand side of Eq. (67), the linear term in $R_{7}$ has the dominant weight. Since in the present scenario $R_{7,8}$ and $\tilde{R}_{7,8}$ are expected to be all of the same order, to simplify the analysis we neglect all the $R_{i}$ terms but the linear term in $R_{7}$ in the rhs of Eq.(67), which will be a fair approximation for the purposes of the present analysis. Then, by requiring that the theoretical central value lies inside the experimental 2- $\sigma$ band of Eq. 68) (with a standard deviation $\sigma=0.22 \times 10^{-4}$ obtained by summing in quadrature the statistical and systematic errors), one obtains the following upper bounds

$$
\begin{aligned}
\left|R_{7}\right| \lesssim 0.139 & \text { for } \operatorname{sign}\left(R_{7}\right)=+1 \\
\left|R_{7}\right| \lesssim 0.071 & \text { for } \operatorname{sign}\left(R_{7}\right)=-1 .
\end{aligned}
$$

Since the $R_{7}$ sign is not predicted in the present framework, we will impose the most conservative upper bounds on $\left|R_{7}\right|<0.139$, corresponding to the positive $R_{7}$ sign. According to the results given in the Appendix, the $R_{7}$ absolute value is given by

$$
\left|R_{7}\right|=\frac{2 \pi^{2} \sqrt{2}}{3 G_{F} V_{32}^{\star} V_{33} \bar{m}_{D}^{2}\left|C_{7}^{S M}\left(M_{W}\right)\right|}\left|\frac{\eta_{L}^{23}}{\eta_{L}^{33}}\right| \bar{F}_{L R}\left(x_{3}^{D}, \xi_{D}\right)
$$

where the expression for the function $\bar{F}_{L R}(x, \xi)$ can be found in Eq. 118$)$ in the Appendix, and $C_{7}^{\mathrm{SM}}\left(M_{W}\right)=-0.193$ for $m_{t}=170 \mathrm{GeV}$ [24]. Then, the constraint $\left|R_{7}\right|<0.139$ sets a lower bound on the effective messenger mass scale $\bar{m}_{D}^{32}$, defined as

$$
\bar{m}_{D}^{32} \equiv \bar{m}_{D} \sqrt{\left|\frac{\eta_{L}^{33}}{\eta_{L}^{23}}\right|},
$$

versus $x_{3}^{D}$ and $\xi_{D}$. In Fig. 4 , we plot the $\bar{m}_{D}^{32}$ regions excluded at $95 \%$ C.L. by $b \rightarrow s \gamma$ data, as a function of $x_{3}^{D}$, and for several values of the $\xi_{D}$ mixing.

If we now combine the DM constraints on $\bar{m}_{D}=\bar{m}_{U}$ in Eq. (47), with the ones from $b \rightarrow s \gamma$ in Fig. 4, we can see that the latter do not allow to set any stringent upper limit on the flavor 


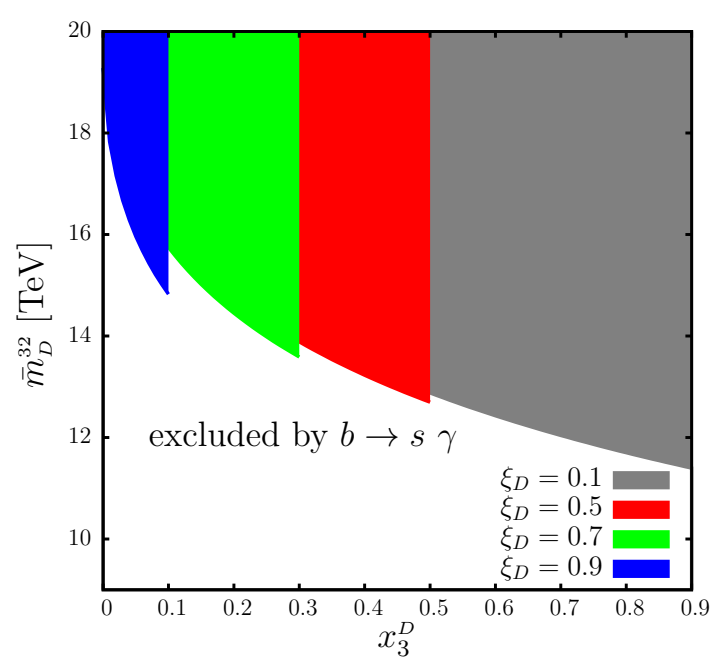

Figure 4: Regions allowed by $b \rightarrow s \gamma$ data at 95\% C.L. (represented by superimposed colored areas), for the effective messenger mass scale $\bar{m}_{D}^{32}$ defined in Eq. (71), as a function of $x_{3}^{D}$ and for different values of the mixing parameter $\xi_{D}$. Regions $x_{3}^{D}>1-\xi_{D}$ are excluded by DM constraints.

matrix elements $\eta_{L}^{23}, \eta_{L}^{33}$, since in this case $\bar{m}_{U}$ would be always inside the allowed regions of $\bar{m}_{D}^{32}$ in Fig. 4 [see Eq. (47)].

On the contrary, in the NUF scenario, lower messenger masses are allowed ( $c f$. Eq.(52)), and strong upper bounds on the combination $\frac{\left|\eta_{L}^{23}\right|}{\left|\eta_{L}^{33}\right|}$ arise from the $b \rightarrow s \gamma$ constraints. For example, combining DM and $b \rightarrow s \gamma$ constraints we get, for $\xi_{D}=0.5$,

$$
\left|\frac{\eta_{L}^{23}}{\eta_{L}^{33}}\right|<7 \times 10^{-2}\left(\frac{\bar{m}_{D}}{3 \mathrm{TeV}}\right)^{2}, \quad \text { if } \quad \bar{m}_{D} \geq 3 \mathrm{TeV} .
$$

We now analyze the $\operatorname{BR}(b \rightarrow s \bar{\gamma})$ upper bounds given by the $b \rightarrow s \gamma$ data in Fig. 4 . For simplicity, we will assume a left-right symmetry, namely $\Lambda_{L}^{b s}=\Lambda_{R}^{b s}$. Then, the $1 /\left(\bar{m}_{D}^{32}\right)^{2}$ scale, defined by Eq. (71), factorizes in both the $b \rightarrow s \gamma$ and $b \rightarrow s \bar{\gamma}$ amplitudes. For the NP contribution saturating the $R_{7}<R_{7}^{\max }=0.139$ bound arising from the $b \rightarrow s \gamma$ measurement, we get then

$$
\frac{1}{\Lambda_{L}^{b s}}<\left(\frac{3 m_{b} \bar{e} \bar{e}_{3}^{D} G_{F} V_{32}^{\star} V_{33}}{2 \pi^{2} \sqrt{2}}\right) \bar{F}_{L R}\left(x_{3}^{D}, \xi_{D}\right) R_{7}^{\max }\left|C_{7}^{\mathrm{SM}}\left(M_{W}\right)\right|
$$

which can be translated into an upper bound on $\operatorname{BR}(b \rightarrow s \bar{\gamma})$. In particular, we obtain, for representative $\xi_{D}$ and $x_{3}^{D}$ values ${ }^{6}$

\footnotetext{
${ }^{6}$ Actually, these bounds are independent from the matrix elements $\eta_{L, R}^{23}$ and $\eta_{L, R}^{33}$ only if we require the left-right universality assumption $\eta_{L}^{j i}=\eta_{R}^{j i}$ or by considering the contribution of each of them at a time, since these can factorize in both $b \rightarrow s \gamma$ and $b \rightarrow s \bar{\gamma}$ amplitudes.
} 
- for $\xi_{D}=0.1$ and $x_{3}^{U}=0.8$ (small-mixing regime)

$$
\mathrm{BR}^{(b \rightarrow s \gamma)}(b \rightarrow s \bar{\gamma})<6.9 \times 10^{-3}\left(\frac{\bar{\alpha}}{0.1}\right),
$$

- for $\xi_{D}=0.8$ and $x_{3}^{U}=0.1$ (large-mixing regime)

$$
\mathrm{BR}^{(b \rightarrow s \gamma)}(b \rightarrow s \bar{\gamma})<1.0 \times 10^{-2}\left(\frac{\bar{\alpha}}{0.1}\right)
$$

where we have set $\bar{e}_{3}^{D}=1$, and used the approximated relation for $x_{3}^{D}$ in Eq.(62). Typical values $\bar{\alpha} \simeq 0.1$ are natural in the present framework [12. In the NUF scenario, where $x_{3}^{U}$ and $x_{3}^{D}$ are independent variables, we get

- for $\xi_{D}=0.1$ and $x_{3}^{D}=0.8$ (small-mixing regime)

$$
\mathrm{BR}^{(b \rightarrow s \gamma)}(b \rightarrow s \bar{\gamma})<2.5 \times 10^{-4}\left(\frac{\bar{\alpha}}{0.1}\right),
$$

- for $\xi_{D}=0.8$ and $x_{3}^{D}=0.1$ (large-mixing regime)

$$
\mathrm{BR}^{(b \rightarrow s \gamma)}(b \rightarrow s \bar{\gamma})<4.8 \times 10^{-4}\left(\frac{\bar{\alpha}}{0.1}\right) .
$$

Notice that these upper bounds are independent from the effective messenger scale $\bar{m}_{D}^{32}$, since the latter has been set to saturate the upper bound on $R_{7}$ coming from the $b \rightarrow s \gamma$ data. In Fig. 5, we show the resulting $\mathrm{BR}(b \rightarrow q \bar{\gamma})$ expectations versus mixing. The blue area corresponds to the allowed ranges, while the red area select the regions excluded by the $\mathrm{BR}(b \rightarrow$ $q \gamma)$ bounds. One can see that large values for $\operatorname{BR}(b \rightarrow q \bar{\gamma})$ are presently allowed, both in the UF (left plot) and NUF (right plot). In particular, for unit couplings, the UF scenario allows $\operatorname{BR}(b \rightarrow q \bar{\gamma})$ 's up to $\left(10^{-8}-10^{-3}\right)$, depending on the mixing value, while the NUF scenario allows up to $\left(10^{-6}-10^{-4}\right)$.

\section{3 $\quad \operatorname{BR}(b \rightarrow q \bar{\gamma})$ upper bounds from $B_{q^{-}} \bar{B}_{q}$ mixing measurements}

In this section we estimate the largest effect induced by the NP contribution to the effective Hamiltonian for the $\Delta B=2$ transitions. Then we will analyze its impact on the $B_{d}-\bar{B}_{d}$ and $B_{s}-\bar{B}_{s}$ mixing measurements.

The effective Hamiltonian for the $\left|\Delta B_{s}\right|=2$ transitions, induced by the Lagrangian in Eq.(19), is given by

$$
H_{e f f}^{\left|\Delta B_{s}\right|=2}=\frac{1}{64 \pi^{2} \bar{m}^{2}(1-\xi)}\left[\sum_{i=1}^{5} C_{i} Q_{i}+\sum_{i=1}^{3} \tilde{C}_{i} \tilde{Q}_{i}\right]+\text { H.c. }
$$



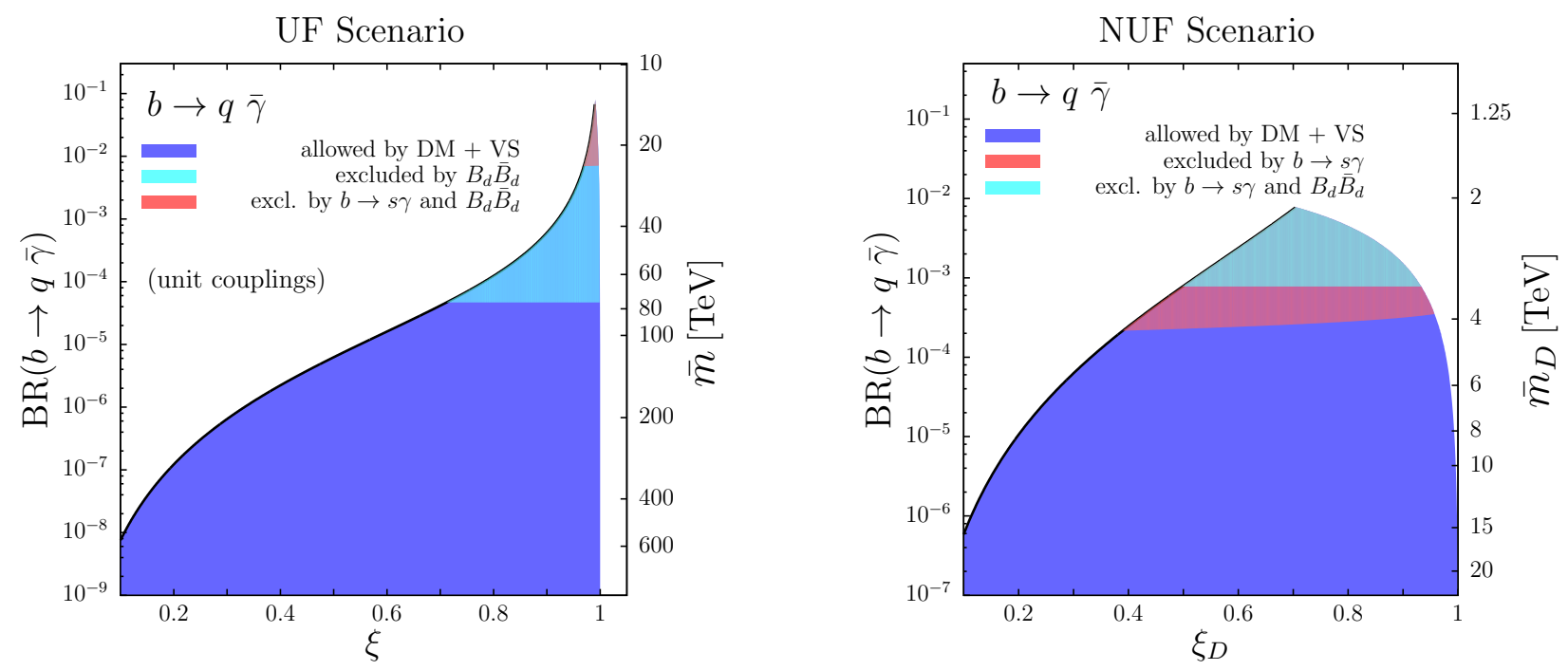

Figure 5: Allowed regions (dark blue colored areas) by DM and vacuum stability (VS) constraints for $\operatorname{BR}(b \rightarrow q \bar{\gamma})$ and for the average messenger mass scales $\bar{m}$ and $\bar{m}_{D}$, versus the corresponding mixing $\xi$ and $\xi_{D}$, in the UF (left) and NUF (right) scenarios, respectively. In the UF (NUF) scenario, we assume $\bar{e} \bar{e}_{3}^{D}=1, \eta_{L}^{j 3} / \eta_{L}^{33}=1(0.1)$, with $j=1,2$. Red regions are excluded by the $b \rightarrow s \gamma$ constraints, and light-blue regions are excluded by both $b \rightarrow s \gamma$ and $B_{d} \bar{B}_{d}$ mixing constraints.

where the operators $Q_{1-5}$ are defined as

$$
\begin{aligned}
Q_{1} & =\left(\bar{b}_{L}^{\alpha} \gamma_{\mu} s_{L}^{\alpha}\right)\left(\bar{b}_{L}^{\beta} \gamma^{\mu} s_{L}^{\beta}\right) \\
Q_{2} & =\left(\bar{b}_{R}^{\alpha} s_{L}^{\alpha}\right)\left(\bar{b}_{R}^{\beta} s_{L}^{\beta}\right) \\
Q_{3} & =\left(\bar{b}_{R}^{\alpha} s_{L}^{\beta}\right)\left(\bar{b}_{R}^{\beta} s_{L}^{\alpha}\right) \\
Q_{4} & =\left(\bar{b}_{R}^{\alpha} s_{L}^{\alpha}\right)\left(\bar{b}_{L}^{\beta} s_{R}^{\beta}\right) \\
Q_{5} & =\left(\bar{b}_{R}^{\alpha} s_{L}^{\beta}\right)\left(\bar{b}_{L}^{\beta} s_{R}^{\alpha}\right)
\end{aligned}
$$

with $\tilde{Q}_{i}=Q_{i}(L \leftrightarrow R)$ and $q_{L, R} \equiv \frac{1}{2}\left(1 \mp \gamma_{5}\right) q$. Also, $q=b, s$ stand for the $b$-quark and $s$ quarks fields, respectively, and $\alpha, \beta$ are color indices (sum over color indices is understood). The operator basis corresponding to the effective Hamiltonian for $\left|\Delta B_{d}\right|=2$ is simply obtained by replacing $s$ with $d$ quark fields in $Q_{i}$ and $\tilde{Q}_{i}$ operators in Eq. 79. .

In order to obtain the Wilson coefficients $C_{i}$ and $\tilde{C}_{i}$, we compute the contributions at one loop to the box diagrams for the process $\bar{b} s \rightarrow b \bar{s}$, by neglecting quark masses and external momenta. Since we are interested to their dominant effect, we will work in the approximation of large mixing $\xi$, which allows us to restrict to the contribution of the Feynman diagrams in which only the two lightest scalars circulate in the loop. In the left-right symmetric scenario considered here, this corresponds to consider in the box diagram only the propagation of two 
degenerate messengers with mass square $m_{-}^{2}=\bar{m}^{2}(1-\xi)$. Since we are interested in constraining only the combination of flavor matrix elements $\eta_{32}$ and $\eta_{31}$ (which enter the $b \rightarrow s \bar{\gamma}$ and $b \rightarrow d \bar{\gamma}$ processes, respectively), in order to simplify the analysis, we will consider only the contribution to the $B_{q}-\bar{B}_{q}$ mixing induced by the dark-fermion associated to the $b$-quark, namely $Q_{D_{3}}$, while we assume for the diagonal entries, $\eta_{33}=1$ and $\eta_{i i}=0$ for $i=1,2$.

By using the above approximations and performing the matching between the amplitude of $\bar{b} s \rightarrow b \bar{s}$ computed from the full theory and the one obtained by the effective Hamiltonian in Eq. (78), we obtain the following results for the Wilson coefficients evaluated at the messenger mass scale $\bar{m}_{-}$:

$$
\begin{aligned}
C_{1} & =\frac{1}{2} C_{L L}^{2} \Delta_{1}, \quad \tilde{C}_{1}=\frac{1}{2} C_{R R}^{2} \Delta_{1}, \\
C_{2} & =\tilde{C}_{2}=0, \\
C_{3} & =\frac{1}{2} C_{R L}^{2} \Delta_{2}, \quad \tilde{C}_{3}=\frac{1}{2} C_{L R}^{2} \Delta_{2}, \\
C_{4} & =-2 C_{L R} C_{R L} \Delta_{1}, \quad C_{5}=C_{L R} C_{R L} \Delta_{2},
\end{aligned}
$$

where the coefficients $C_{L, R}$ are defined as

$$
\begin{array}{ll}
C_{L L}=g_{L}^{2} \eta_{L}^{3 j}\left(\eta_{L}^{j 2}\right)^{\star}, & C_{R R}=g_{R}^{2} \eta_{R}^{3 j}\left(\eta_{R}^{j 2}\right)^{\star}, \\
C_{L R}=g_{L} g_{R} \eta_{L}^{3 j}\left(\eta_{R}^{j 2}\right)^{\star}, & C_{R L}=g_{L} g_{R} \eta_{R}^{3 j}\left(\eta_{L}^{j 2}\right)^{\star}
\end{array}
$$

and $j=3$ in case one considers only the exchange of the $Q_{D_{3}}$ dark-fermion. As for the quantities $\Delta_{1,2}$, which parametrize the loop integrals, we get the following results for the UF and NUF scenarios

$$
\begin{gathered}
\Delta_{1}^{U F}=-\frac{1}{4}, \quad \Delta_{2}^{U F}=0, \\
\Delta_{1}^{N U F}=-\frac{1}{12}, \quad \Delta_{2}^{N U F}=\frac{1}{6} .
\end{gathered}
$$

In the UF scenario, the loop integrals in Eq. (82) have been obtained by setting to zero the dark-fermion mass, which is well justified since in this case the average messenger mass is much larger than $M_{Q_{D_{3}}}$. On the other hand, in the NUF scenario, we have retained the contribution of the dark-fermion mass of third generation $M_{Q_{D_{3}}}$ and set it equal to the lightest messenger mass $M_{Q_{D_{3}}}^{2} \simeq \bar{m}^{2}(1-\xi)$, as assumed in the the NUF scenario contribution to $B R(b \rightarrow q \bar{\gamma})$ in order to pinpoint the largest effect. Regarding the effective Hamiltonian for the $\left|\Delta B_{d}\right|=2$ transitions, the corresponding Wilson coefficients can be obtained by the $C_{i}$ and $\tilde{C}_{i}$ expressions above, by replacing in Eq. 81) the $\eta_{L, R}^{j 2}$ matrix elements by $\eta_{L, R}^{j 1}$, with $j=3$.

The contribution to the $B_{q}-\bar{B}_{q}$ mixing amplitude $M_{12}^{q}$ is given by

$$
M_{12}^{q}=\frac{\left\langle B_{q}\left|H_{e f f}^{\left|\Delta B_{q}\right|=2}\right| \bar{B}_{q}\right\rangle}{2 M_{B_{q}}}
$$

where $M_{B_{q}}$ is the neutral $B$-meson mass, with $q=d, s$. Combining the SM with the NP contributions, one obtains for the difference of the neutral $\mathrm{B}$ meson mass eigenstates system 
$\Delta M_{q}=M_{H}^{q}-M_{L}^{q}=2\left|M_{12}^{q}\right|$, where $M_{H}^{q}$ and $M_{L}^{q}$ are the corresponding heavy and light mass eigenstates of the neutral $B_{q}-\bar{B}_{q}$ system respectively, [33]

$$
\begin{aligned}
\Delta M_{d} & =0.502 \mathrm{ps}^{-1}\left|\Delta_{d}\right|, \\
\Delta M_{s} & =17.24 \mathrm{ps}^{-1}\left|\Delta_{s}\right|,
\end{aligned}
$$

where $z_{t}=\frac{\bar{m}_{t}^{2}}{M_{W}^{2}}$, and the $\Delta_{q}$ quantities are defined as

$$
\Delta_{q} \equiv 1+\frac{M_{12}^{N P, q}}{M_{12}^{S M, q}} .
$$

Above, $M_{12}^{N P, q}\left(M_{12}^{S M, q}\right)$ stands for the NP (SM) corresponding contribution. In the above Eq. 84), we assume the central values reported in [33], in particular $\left|V_{t b} V_{t d}^{\star}\right|=0.0086,\left|V_{t b} V_{t s}^{\star}\right|=$ $0.04, f_{B_{d}}^{2} B_{B_{d}}=(0.17 \mathrm{GeV})^{2}, f_{B_{s}}^{2} B_{B_{s}}=(0.21 \mathrm{GeV})^{2}$, and $S\left(\frac{\bar{m}_{t}^{2}}{M_{W}^{2}}\right)=2.35$, where $S(x)$ is the Inami-Lim function for the top-quark contribution from the box diagram, $\bar{m}_{t}$ the top-quark mass in MS scheme evaluated at $\bar{m}_{t}$ scale $\left[\bar{m}_{t}\left(\bar{m}_{t}\right)=0.957 m_{t}\right], f_{B_{q}}$ the $B_{q}$ decay constants, and $B_{B_{q}}$ the bag factors related to the matrix element of the corresponding $\Delta B=2 \mathrm{SM}$ operators.

We then computed the Wilson coefficients at the low energy scale of order $\mathcal{O}\left(m_{b}\right)$, and the matrix elements of the operators appearing in Eq.(79) at the NLO and evaluated at the same scale, by using the results of [34, where the same structure for the effective Hamiltonian was considered. Following the results of [35], the present $B_{q}-\bar{B}_{q}$ mixing measurements imply

$$
\operatorname{Re}\left(\Delta_{d}\right)=0.823_{-0.095}^{+0.143}, \quad \operatorname{Re}\left(\Delta_{s}\right)=0.965_{-0.078}^{+0.133}
$$

where corresponding errors are at 1- $\sigma$ level. Assuming a constructive NP contribution to the SM result (and real $\eta$ matrices), where the NP contribution to $\left(\left|\operatorname{Re}\left(\Delta_{d}\right)\right|-1\right)$ is more constrained, we require $\left(\left|\operatorname{Re}\left(\Delta_{q}\right)\right|-1\right)$ to lie at the $2-\sigma$ level in the following ranges

$$
0 \leq\left|\operatorname{Re}\left(\Delta_{d}\right)\right|-1<0.109, \quad 0 \leq\left|\operatorname{Re}\left(\Delta_{s}\right)\right|-1<0.231 .
$$

In Fig. 5, we show the effect of the $B_{q}-\bar{B}_{q}$ mixing constraints on $\operatorname{BR}(b \rightarrow q \bar{\gamma})$ versus $\xi$, for the UF (left plot) and NUF (right plot) scenarios. The light-blue areas are the excluded ones. We focus on the $B_{d^{-}} \bar{B}_{d}$ mixing constraints [which hold for the $\mathrm{BR}(b \rightarrow d \bar{\gamma})$ case], since the regions excluded by the $B_{s}-\bar{B}_{s}$ mixing are always outside the area allowed by DM and $b \rightarrow s \gamma$ constraints. One can see that the $B_{d^{-}} \bar{B}_{d}$ mixing is quite effective, disfavoring $\operatorname{BR}(b \rightarrow d \bar{\gamma})$ values above $5 \times 10^{-5}$ and $8 \times 10^{-4}$ for the UF and NUF scenarios, respectively.

\section{$6 \quad$ The $c \rightarrow u \bar{\gamma}$ decay}

Here we analyze the FCNC decay $c \rightarrow u \bar{\gamma}$, following the same approach as used for the heavier quarks. The corresponding total width is given by Eq.(39), where $i=2$ and $j=1$ for the $c \rightarrow u$ transition. We will express $\mathrm{BR}(c \rightarrow u \bar{\gamma})$ in terms of the inclusive decay rate $\mathrm{BR}^{\exp }(c \rightarrow$ $\left.\ell^{+} X\right)=(0.096 \pm 0.004) \%$ (with $X$ standing for anything) [14], by approximating $\Gamma\left(c \rightarrow \ell^{+} X\right)$ with the Cabibbo-allowed tree-level $c \rightarrow s e^{+} \nu_{e}$ decay width. 


\begin{tabular}{|c||c|c|}
\hline$\xi$ & $\mathrm{BR}_{\mathrm{UF}}^{\max }(c \rightarrow u \bar{\gamma})$ & $\mathrm{BR}_{\mathrm{NUF}}^{\max }(c \rightarrow u \bar{\gamma})$ \\
\hline 0.1 & $1.0 \times 10^{-11}$ & $2.9 \times 10^{-13}$ \\
\hline 0.2 & $1.6 \times 10^{-10}$ & $4.9 \times 10^{-12}$ \\
\hline 0.3 & $8.5 \times 10^{-10}$ & $2.8 \times 10^{-11}$ \\
\hline 0.5 & $8.1 \times 10^{-9}$ & $3.8 \times 10^{-10}$ \\
\hline 0.7 & $5.3 \times 10^{-8}$ & $5.1 \times 10^{-9}$ \\
\hline 0.8 & $1.6 \times 10^{-7}$ & $2.9 \times 10^{-8}$ \\
\hline 0.9 & $7.5 \times 10^{-7}$ & $4.9 \times 10^{-7}$ \\
\hline 0.95 & $3.2 \times 10^{-6}$ & $7.7 \times 10^{-6}$ \\
\hline
\end{tabular}

Table 6: Maximum values of $\mathrm{BR}(c \rightarrow u \bar{\gamma})$ allowed by vacuum stability and DM constraints versus the mixing parameter $\xi=\xi_{U}=\xi_{D}$ and $\xi=\xi_{D}$, in the UF and NUF scenarios, respectively. Results are in unit of couplings, that is they assume $\bar{e} \bar{e}_{2}^{U}=g_{L, R}=\rho_{L, R}^{12,22}=\eta_{L, R}^{12,22}=1$, with all other elements of flavor matrices set to zero.

Then, one has

$$
\operatorname{BR}(c \rightarrow u \bar{\gamma})=\frac{12 \mathrm{BR}^{\exp }\left(c \rightarrow \ell^{+} X\right)}{G_{F}^{2}\left|V_{c s}\right|^{2} m_{c}^{2} f_{1}\left(z_{u c}\right)}\left(\frac{1}{\left(\Lambda_{L}^{c u}\right)^{2}}+\frac{1}{\left(\Lambda_{R}^{c u}\right)^{2}}\right)
$$

where $V_{c s}$ is the relevant CKM matrix element, and $z_{u c}=m_{u}^{2} / m_{c}^{2}$, with $f_{1}(x)$ defined by Eq.(60). The expressions needed for $\Lambda_{L, R}^{c u} \equiv\left(\Lambda_{L, R}^{U}\right)_{21}$ can be found in Eq. (29). For our numerical analysis, we use the central value of $V_{c s}=0.986 \pm 0.016$, extracted from the average of the $D$ leptonic and semileptonic decays [14].

Following the same strategy as the one described for the top and $b$-quark cases, we report in Table 6 the results for the maximum allowed value of $\operatorname{BR}(c \rightarrow u \bar{\gamma})$, satisfying the vacuum stability bounds and DM constraints versus the mixing parameter $\xi=\xi_{U}=\xi_{D}\left(\xi=\xi_{D}\right)$ in the UF (NUF) scenario. These results assume $U(1)_{F}$ charges and other multiplicative couplings normalized to 1 . In particular, in Table 6 one has $\bar{e} \bar{e}_{2}^{U}=g_{L, R}=\rho_{L, R}^{12,22}=\eta_{L, R}^{12,22}=1$, with all other elements of flavor matrices set to zero.

Finally, in Fig. 6, we show the corresponding regions of $\mathrm{BR}(c \rightarrow q \bar{\gamma})$ values allowed by DM and vacuum stability constraints versus the mixing parameter. The blue area corresponds to the allowed ranges. Experimental upper bounds on $\operatorname{BR}(c \rightarrow q \gamma)$ do not further constraint the blue regions in this case. One can see that large values for $\operatorname{BR}(c \rightarrow q \bar{\gamma})$ are presently allowed, both in the UF (left plot) and NUF (right plot). In particular, for unit couplings, the UF scenario allows $\operatorname{BR}(c \rightarrow q \bar{\gamma})$ 's up to $\left(10^{-11}-10^{-4}\right)$, depending on the mixing value, while the NUF scenario allows up to $\left(10^{-13}-10^{-8}\right)$.

\section{$7 \quad$ The $\tau \rightarrow(\mu, e) \bar{\gamma}$ decays}

We now consider the extension of the model described in Sec. 2 to the leptonic sector in order to also generate effective lepton Yukawa couplings. Notice that we will not include the possibility of Majorana masses neither at tree level nor radiatively generated for the neutrino sector, and 

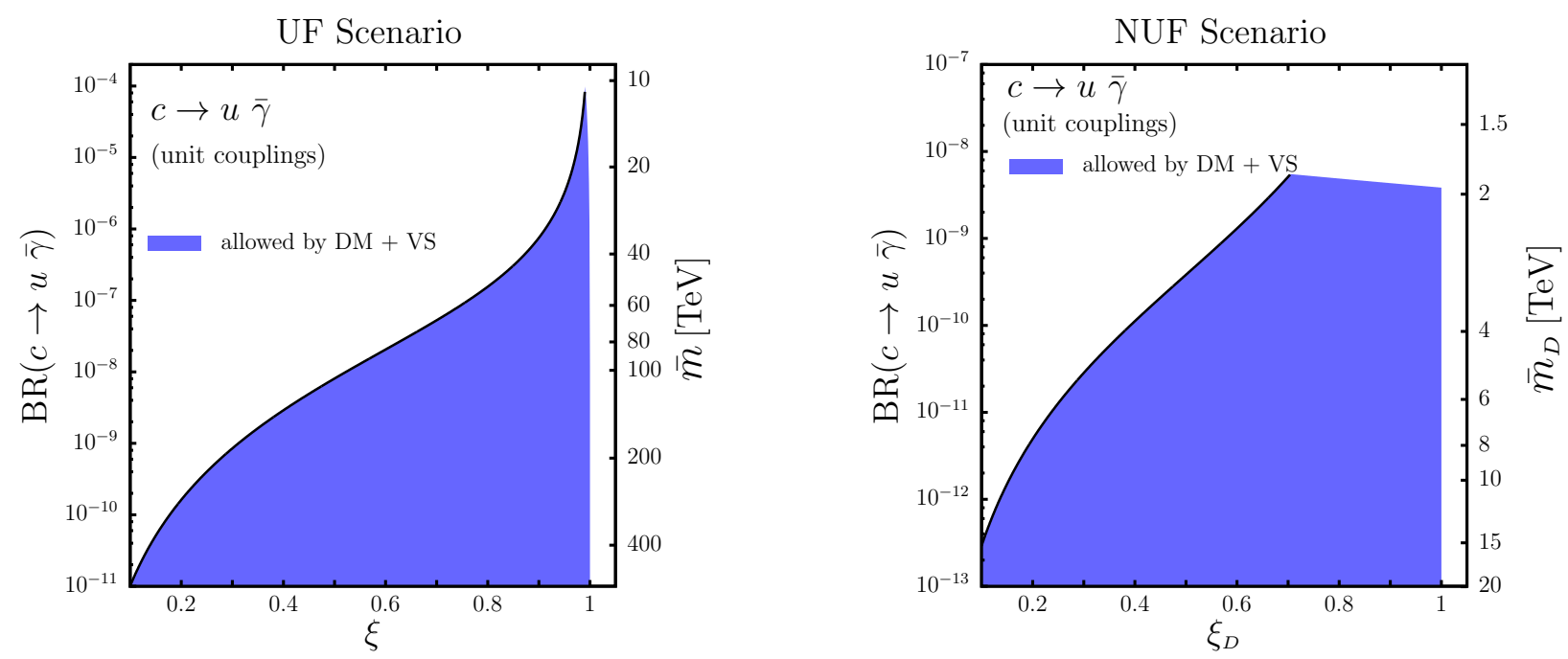

Figure 6: Allowed regions (colored areas) by DM and vacuum-stability (VS) constraints for $\mathrm{BR}(c \rightarrow u \bar{\gamma})$ and for the average messenger mass scales $\bar{m}$ and $\bar{m}_{D}$ versus the corresponding mixing $\xi$ and $\xi_{D}$, in the UF (left) and NUF (right) scenarios, respectively. In the left (right) plots we assume $\bar{e} \bar{e}_{2}^{U}=1, \rho_{L}^{12} / \rho_{L}^{22} \simeq 1\left(\bar{e} \bar{e}_{2}^{D}=1, \eta_{L}^{12} / \eta_{L}^{22} \simeq 1\right)$ with all other matrix elements of flavor matrices set to zero.

neutrinos will be assumed to acquire only a Dirac mass through the SM Higgs mechanism. Although one can also radiatively generate Majorana masses in this framework, we will not consider this possibility here.

New dark fermions will be associated to the charged leptons and neutrinos, as occurs in the quark scenario, with a corresponding set of color singlet messenger fields, having the same $S U(2)_{L} \times U(1)_{Y}$ quantum numbers of the ones related to the lepton sector. Moreover, in order to generate the Pontecorvo-Maki-Nakagawa-Sakata (PMNS) [36] lepton mixing matrix, we will generalize the mechanism described in Sec. 2.3 for the origin of the CKM matrix to the leptonic sector. The induced PMNS matrix will be also unitary, since we will not include any seesaw mechanism.

The generalization to the leptonic sector of the interaction Lagrangian in Eq. (19) is straightforward, consisting just in the substitution of quark messenger and dark-fermion fields by the corresponding ones in the leptonic sector. We then just provide the notation for the new flavor mixing matrices in the leptonic sector. In particular, after rotating the lepton fields to the masseigenstate basis, new flavor matrices $\tilde{\rho}, \tilde{\eta}$ will appear in the leptonic Lagrangian corresponding to Eq. (19), where

$$
\begin{aligned}
\rho_{L, R} & \rightarrow \tilde{\rho}_{L, R} \\
\eta_{L, R} & \rightarrow \tilde{\eta}_{L, R} .
\end{aligned}
$$

In this framework, we first analyze the flavor-violating tau lepton decays

$$
\tau \rightarrow \ell \bar{\gamma}
$$


where $\ell=\mu, e 7$. The corresponding decay width can be inferred by Eq. (39), with $i=3$ and $j=2,1$ for the $\ell=\mu$,e transitions, respectively. The $\Lambda_{L, R}^{\tau \mu}$ and $\Lambda_{L, R}^{\tau e}$ expressions can be obtained from $\left(\Lambda_{L, R}^{D}\right)_{32}$ and $\left(\Lambda_{L, R}^{D}\right)_{31}$ as defined in Eq. (30), where the quark masses in the down sector $m_{D_{i}}$, the dark-quark masses $M_{Q_{i}^{U, D}}$, and average messenger masses $\bar{m}_{U, D}$ are replaced by the corresponding ones in the leptonic sector, namely $m_{E_{i}}, M_{L_{i}^{U, D}}, \bar{m}_{L}^{U, D}$, respectively. In Eq. 30 , one then makes the replacements $\left(x_{3}^{D}, \xi_{D}\right) \rightarrow\left(x_{3}^{L}, \xi_{L}\right)$, where $x_{3}^{L} \equiv\left(M_{L_{3}^{D}} / \bar{m}_{L}^{D}\right)^{2}$, and $g_{L, R} \rightarrow \bar{g}_{L, R}$, where $\bar{g}_{L, R}$ are the relevant couplings in the leptonic sector. As for the flavor matrices, Eq. (89) applies.

We can now express $\operatorname{BR}(\tau \rightarrow \ell \bar{\gamma})$ by normalizing it to $\operatorname{BR}^{\exp }\left(\tau \rightarrow \nu_{\tau} \bar{\nu}_{\mu} \mu\right)=(17.41 \pm$ $0.04) \%$ [14], assuming the following $\tau \rightarrow \mu \nu_{\tau} \bar{\nu}_{\mu}$ tree-level decay width

$$
\Gamma_{0}^{\tau}=\frac{G_{F}^{2} m_{\tau}^{5}}{192 \pi^{3}} f_{1}\left(z_{\mu \tau}\right),
$$

where the function $f_{1}(x)$ is defined just after Eq. $(60)$, and $z_{\mu \tau}=m_{\mu}^{2} / m_{\tau}^{2}$. Then, one obtains

$$
\operatorname{BR}(\tau \rightarrow \ell \bar{\gamma})=\frac{12 \mathrm{BR}_{\tau \rightarrow \nu_{\tau} \bar{\nu}_{\mu} \mu}^{\exp }}{G_{F}^{2} m_{\tau}^{2} f_{1}\left(z_{\mu \tau}\right)}\left(\frac{1}{\left(\Lambda_{L}^{\tau \ell}\right)^{2}}+\frac{1}{\left(\Lambda_{R}^{\tau \ell}\right)^{2}}\right) .
$$

We will restrict to the UF scenario, where the average messenger masses for the up and down $S U(2)_{L}$ messenger fields in the leptonic sector are assumed to be the same, namely $\bar{m}_{L}^{U}=\bar{m}_{L}^{D} \equiv \bar{m}_{L}$. Moreover, in $\Lambda_{L, R}^{\tau \ell}$ we will neglect the terms proportional to $\bar{g}_{L}^{2} /\left(16 \pi^{2}\right)$ (cf. Eq. (30)).

Regarding the constraints coming from DM and vacuum stability, in the leptonic sector the bounds in Eq. (49) reads

$$
\bar{m}_{L} \geq m_{\tau}\left(\frac{16 \pi^{2}}{\bar{g}_{L} \bar{g}_{R}}\right) F\left(\xi_{L}\right)
$$

where $\xi_{L}$ is the universal mixing parameter for the leptonic messenger masses. Then, at large $\xi_{L}$, one has

$$
\bar{m}_{L} \gtrsim 1.1 \sqrt{1-\xi_{L}} \mathrm{TeV} .
$$

The corresponding maximum allowed $\operatorname{BR}(\tau \rightarrow \ell \bar{\gamma})$ is reported in Table 7, where all relevant couplings are set to 1 .

Radiative lepton-flavor violating (LFV) decays $\tau^{-} \rightarrow \ell^{-} \gamma$, with $\ell=\mu, e$, indirectly constrain $\tau$ decays into dark photons. The present experimental upper bounds at 90\% C.L. are [38]

$$
\begin{aligned}
\operatorname{BR}\left(\tau^{-} \rightarrow e^{-} \gamma\right) & <3.3 \times 10^{-8} \\
\operatorname{BR}\left(\tau^{-} \rightarrow \mu^{-} \gamma\right) & <4.4 \times 10^{-8}
\end{aligned}
$$

The SM contribution to the LFV $\tau \rightarrow \ell \gamma$ decays is negligible, due to the GIM suppression and tiny neutrino masses, even accounting for the PMNS matrix. However, the NP contribution could be potentially quite large. In the present scenario the corresponding prediction is

$$
\operatorname{BR}(\tau \rightarrow \ell \gamma)=\frac{12 \mathrm{BR}_{\tau \rightarrow \nu_{\tau} \bar{\nu}_{\mu} \mu}^{\exp }}{G_{F}^{2} m_{\tau}^{2} f_{1}\left(z_{\mu \tau}\right)}\left(\frac{1}{\left(\bar{\Lambda}_{L}^{\tau \ell}\right)^{2}}+\frac{1}{\left(\bar{\Lambda}_{R}^{\tau \ell}\right)^{2}}\right)
$$

\footnotetext{
${ }^{7}$ Flavor violating $\tau$ decays into a massive neutral vector have been considered in 37 .
} 


\begin{tabular}{|c||c|c|c|}
\hline$\xi_{L}$ & $\mathrm{BR}^{\max }(\tau \rightarrow \ell \bar{\gamma})$ & $\bar{m}_{L}^{\min }[\mathrm{TeV}]$ & $m_{L_{-}}^{\min }[\mathrm{TeV}]$ \\
\hline 0.05 & $2.3 \times 10^{-7}$ & 11 & 11 \\
\hline 0.1 & $3.8 \times 10^{-6}$ & 5.7 & 5.4 \\
\hline 0.2 & $6.9 \times 10^{-5}$ & 2.9 & 2.6 \\
\hline$(0.3)$ & $4.1 \times 10^{-4}$ & 1.9 & 1.6 \\
\hline$(0.4)$ & $1.6 \times 10^{-3}$ & 1.4 & 1.1 \\
\hline$(0.5)$ & $5.2 \times 10^{-3}$ & 1.1 & 0.8 \\
\hline
\end{tabular}

Table 7: Maximum values of $\operatorname{BR}(\tau \rightarrow \ell \bar{\gamma})$ allowed by vacuum stability and DM constraints in the UF scenario for the leptonic sector, corresponding to the minimum allowed average mass $\bar{m}_{L}^{\min }$, and to the lightest universal messenger mass eigenvalue $m_{L_{-}}^{\min }=\bar{m}^{\min } \sqrt{1-\xi_{L}}$ versus the mixing parameter $\xi_{L}$. Results are in unit of couplings, that is they assume $\bar{e} \bar{e}_{3}^{L}=\bar{g}_{L, R}=$ $\tilde{\eta}_{L, R}^{33,13,23}=1$, with all other elements of flavor matrices set to zero. BR's corresponding to values of $\xi_{L} \gtrsim 0.2$ might be excluded at $90 \%$ C.L. by direct constraints on $\operatorname{BR}(\tau \rightarrow \ell \gamma)$ (see text).

where the expressions for $\bar{\Lambda}_{L, R}^{\tau \ell}$ can be derived from the general formulas in the Appendix, by replacing the $\eta_{L, R}$ matrices according to Eq. (89), and the variables $\left(x_{3}^{D}, \xi_{D}\right)$ by $\left(x_{3}^{L}, \xi_{L}\right)$.

As discussed above for $b$ decays, a characteristic effective messenger mass scale $1 /\left(\bar{m}_{L}^{32}\right)^{2}$ given by

$$
\bar{m}_{L}^{32} \equiv \bar{m}_{L} \sqrt{\left|\frac{\tilde{\eta}_{L}^{33}}{\tilde{\eta}_{L}^{j 3}}\right|},
$$

with $j=2$ and 1 for $\mu$ and $e$ final states, respectively, factorizes in both $\tau \rightarrow \ell \gamma$ and $\tau \rightarrow \ell \bar{\gamma}$ BR's. Then, the bounds in Eq. (95) can be straightforwardly converted into lower bounds on the effective mass scale $\bar{m}_{L}^{32}$

$$
\bar{m}_{L}^{32}>\left(\frac{96 \pi \alpha \mathrm{BR}_{\tau \rightarrow \nu_{\tau} \bar{\nu}_{\mu} \mu}^{\exp }}{\mathrm{BR}_{\ell \gamma}^{\max } G_{F}^{2} f_{1}\left(z_{\mu \tau}\right)}\right)^{1 / 4} \sqrt{\bar{F}_{L R}\left(x_{3}^{L}, \xi_{L}\right)},
$$

where $\mathrm{BR}_{\ell \gamma}^{\max } \equiv 4.4(3.3) \times 10^{-8}$ for $\ell=\mu(e)$.

In Fig. 7 we plot the excluded regions for $\bar{m}_{L}^{32}$ corresponding to the constraint in Eq. (98), versus $x_{3}^{L}$ and for some values of the mixing parameter $\xi_{L}$. One can see that the constraints depend on $x_{3}^{L}$, with $\bar{m}_{L}^{32} \lesssim(6.7-11.3) \mathrm{TeV}$ in the region $x_{3}^{L}<1-\xi_{L}$, for $\xi_{L} \gtrsim 0.1$.

Analogous results for the constraints from $\tau \rightarrow e \gamma$ can be simply rescaled from the ones in Fig. 7, by using the corresponding upper bound in Eq.95).

As we can see from the results in Fig. 7, the constraints from $\tau \rightarrow \mu \gamma$ or $\tau \rightarrow e \gamma$ on the effective scale $\bar{m}_{L}^{32}$ are a more relaxed than the corresponding ones from $b \rightarrow s \gamma$, for same values of $x_{3}^{D}, \xi_{D}$ and $x_{3}^{L}, \xi_{L}$ (see Fig. 4 for comparison). The reason is that the $b \rightarrow s \gamma$ decay gets the main contribution from the SM, and the constraints apply mainly on the interference between the SM and NP amplitude. On the other hand, for the $\tau \rightarrow \ell \gamma$ decay, the SM contribution is negligible and the constraints apply directly on the new physics contributions to the amplitude squared. 


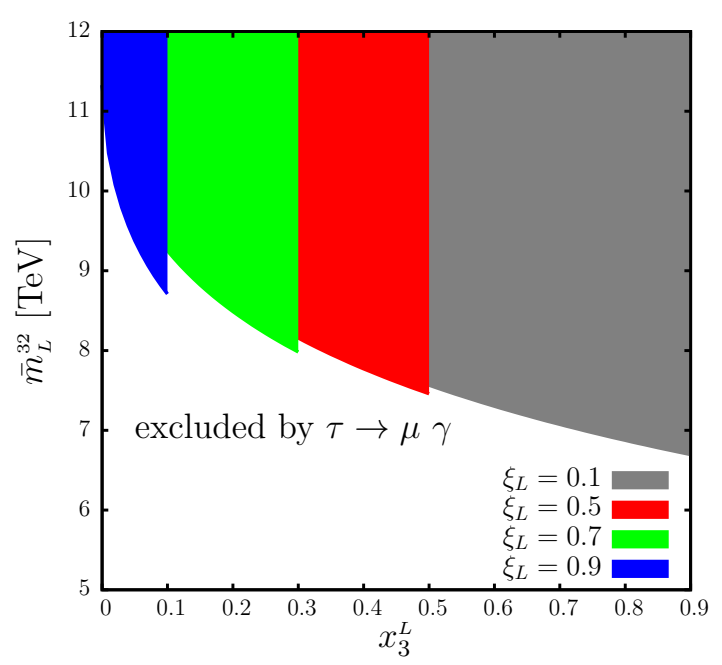

Figure 7: Regions allowed by constraints on $\operatorname{BR}(\tau \rightarrow \mu \gamma)$ at $90 \%$ C.L. (represented by superimposed colored areas), for the effective messenger mass scale $\bar{m}_{L}^{32}$ defined in Eq.(97), as a function of $x_{3}^{L}$ and for several values of the mixing $\xi_{L}$ parameter. Regions $x_{3}^{L}>1-\xi_{L}$ are excluded by DM constraints.

Now, we combine the constraints from $\tau \rightarrow \ell \gamma$ decay with the corresponding ones from DM and vacuum stability. If we compare the values of $\bar{m}_{L}^{\min }$ in Table 7 with the excluded regions in Fig. 7), we can see that no significant upper limits on the mixing matrices $\tilde{\eta}_{L, R}$ can be set at small mixing, since the lower bounds from DM constraints on the average mass $\bar{m}_{L}$ are always above the regions excluded by experimental constraints on $\operatorname{BR}(\tau \rightarrow \ell \gamma)$. On the other hand, for large mixing, the DM constraints are relaxed, and we obtain for example

$$
\begin{aligned}
& \left|\frac{\tilde{\eta}_{L}^{23}}{\tilde{\eta}_{L}^{33}}\right|<1.3 \times 10^{-2}\left(\frac{\bar{m}_{L}}{870 \mathrm{GeV}}\right)^{2}, \quad \text { for } \xi_{L}=0.6, \\
& \left|\frac{\tilde{\eta}_{L}^{23}}{\tilde{\eta}_{L}^{33}}\right|<2.7 \times 10^{-3}\left(\frac{\bar{m}_{L}}{360 \mathrm{GeV}}\right)^{2}, \quad \text { for } \xi_{L}=0.9 .
\end{aligned}
$$

Finally, we give below the upper bounds on $\operatorname{BR}(\tau \rightarrow \ell \bar{\gamma})$ which satisfy the $\tau \rightarrow \ell \gamma$ constraints. In particular, for small and large mixing values we get

- for $\xi_{L}=0.1$ and $x_{3}^{L}=0.8$ (small-mixing regime)

$$
\begin{aligned}
\mathrm{BR}^{(\tau \rightarrow \mu \gamma)}(\tau \rightarrow \mu \bar{\gamma}) & <2.6 \times 10^{-6}\left(\frac{\bar{\alpha}}{0.1}\right) \\
\mathrm{BR}^{(\tau \rightarrow e \gamma)}(\tau \rightarrow e \bar{\gamma}) & <2.0 \times 10^{-6}\left(\frac{\bar{\alpha}}{0.1}\right)
\end{aligned}
$$

- for $\xi_{L}=0.8$ and $x_{3}^{L}=0.1$ (large-mixing regime)

$$
\begin{aligned}
\mathrm{BR}^{(\tau \rightarrow \mu \gamma)}(\tau \rightarrow \mu \bar{\gamma}) & <5.1 \times 10^{-6}\left(\frac{\bar{\alpha}}{0.1}\right) \\
\mathrm{BR}^{(\tau \rightarrow e \gamma)}(\tau \rightarrow e \bar{\gamma}) & <3.8 \times 10^{-6}\left(\frac{\bar{\alpha}}{0.1}\right)
\end{aligned}
$$



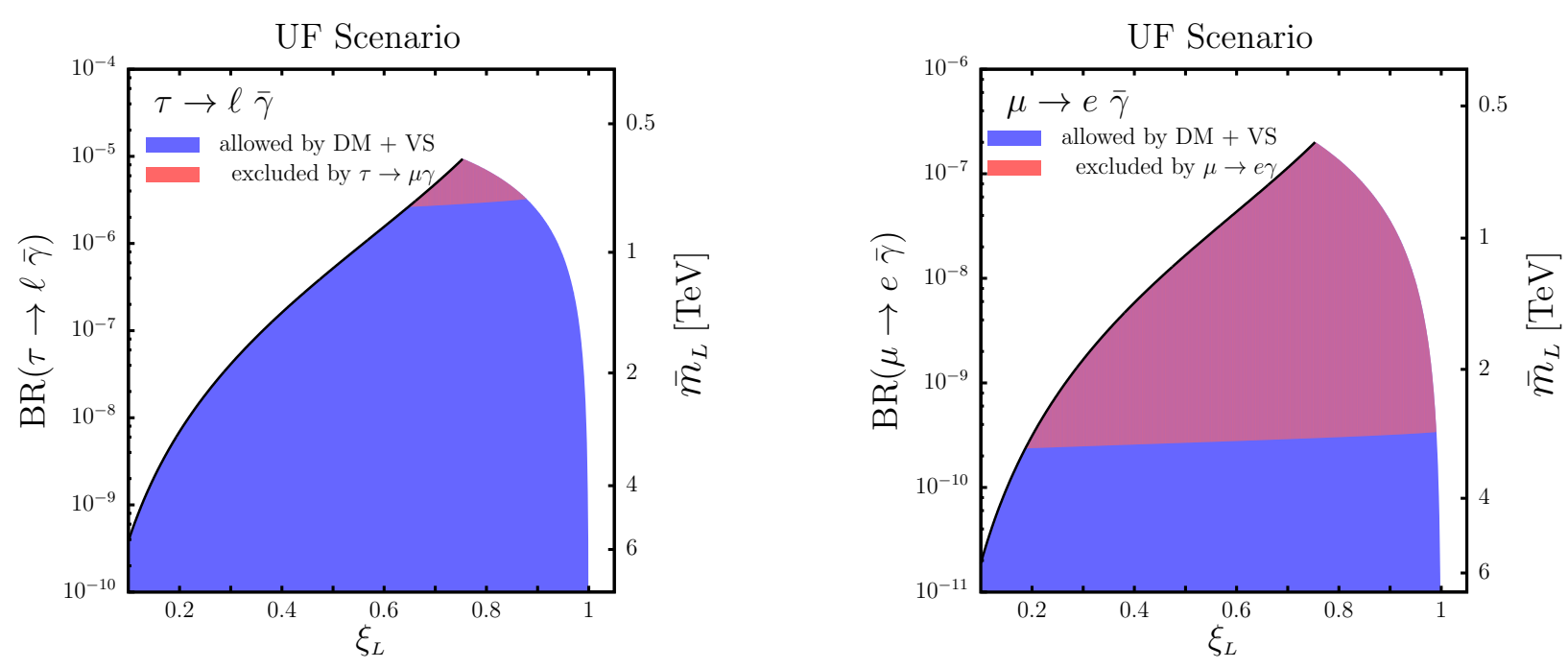

Figure 8: Regions allowed by DM and vacuum stability (VS) constraints for $\operatorname{BR}(\tau \rightarrow \ell \bar{\gamma}$ ) (left) and $\operatorname{BR}(\mu \rightarrow e \bar{\gamma})$ (right), and for the average messenger mass scale $\bar{m}_{L}$, versus the mixing $\xi_{L}$, in the UF scenario (blue areas). Superimposed red areas are the subregions excluded by direct constraints on $\operatorname{BR}\left(\ell \rightarrow \ell^{\prime} \gamma\right)$. In the left (right) plot, we assume $\bar{e} \bar{e}_{3}^{L}=1, \tilde{\eta}_{L}^{j 3} / \tilde{\eta}_{L}^{33}=10^{-2}$ $\left(\bar{e} \bar{e}_{3}^{L}=1, \tilde{\eta}_{L}^{12} / \tilde{\eta}_{L}^{22}=10^{-4}\right)$, with $j=1,2$.

In Fig. 8 (left plot), we show the resulting $\operatorname{BR}(\tau \rightarrow \ell \bar{\gamma})$ expectations versus mixing, in the UF scenario. The blue area corresponds to the allowed ranges, while the red area selects the subregions excluded by the $\operatorname{BR}(\tau \rightarrow \mu \gamma)$ bounds. One can see that, for $\bar{e} \bar{e}_{3}^{L}=1, \tilde{\eta}_{L}^{j 3} / \tilde{\eta}_{L}^{33}=10^{-2}$ (with $j=1,2), \operatorname{BR}(\tau \rightarrow \ell \bar{\gamma})$ 's up to $\left(10^{-10}-10^{-6}\right)$ are allowed, depending on mixing.

\section{The $\mu \rightarrow e \bar{\gamma}$ decay}

Here we analyze the radiative LFV muon decay

$$
\mu \rightarrow e \bar{\gamma},
$$

following the analysis done for the LFV $\tau$ decay into a dark photon. As for the $\tau$ lepton, the corresponding BR can be parametrized in terms of the tree-level $\mathrm{BR}\left(\mu \rightarrow \nu_{\mu} \bar{\nu}_{e} e\right)$, as follows

$$
\mathrm{BR}(\mu \rightarrow e \bar{\gamma})=\frac{12 \mathrm{BR}_{\mu \rightarrow \nu_{\mu} \bar{\nu}_{e} e}^{\exp }}{G_{F}^{2} m_{\mu}^{2} f_{1}\left(z_{e \mu}\right)}\left(\frac{1}{\left(\Lambda_{L}^{\mu e}\right)^{2}}+\frac{1}{\left(\Lambda_{R}^{\mu e}\right)^{2}}\right)
$$

where notations are defined in the previous section, and $\mathrm{BR}^{\exp }\left(\mu \rightarrow \nu_{\mu} \bar{\nu}_{e} e\right) \simeq 100 \%$ [14. As in Eq. (97), we define an effective messenger mass $\bar{m}_{L}^{21}$ given by

$$
\bar{m}_{L}^{21} \equiv \bar{m}_{L} \sqrt{\left|\frac{\tilde{\eta}_{L}^{22}}{\tilde{\eta}_{L}^{12}}\right|} .
$$

which factorizes in the BR if we require the $L$ - $R$ symmetry by assuming $\bar{g}_{L}=\bar{g}_{R}$. The maximum allowed $\operatorname{BR}(\mu \rightarrow e \bar{\gamma})$ by DM and vacuum stability constraints are reported in Fig. 8 (right plot), 


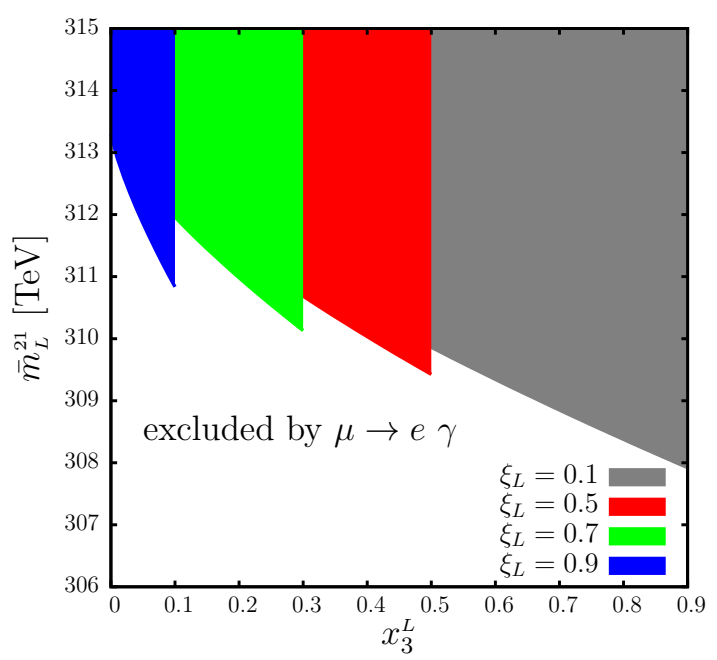

Figure 9: Regions allowed by $\mu \rightarrow e \gamma$ constraints at 90\% C.L. (represented by superimposed colored areas), for the effective messenger mass scale $\bar{m}_{L}^{21}$ defined in Eq.(106), as a function of $x_{3}^{L}$ and for different values of the mixing $\xi_{L}$. Regions $x_{3}^{L}>1-\xi_{L}$ are excluded by DM constraints.

where we also report the constraints due to the LFV $\mu \rightarrow e \gamma$ decay. The present experimental upper bound at $90 \%$ C.L. has been recently obtained by the MEG experiment at the Paul Scherrer Institute [39]

$$
\operatorname{BR}^{\exp }(\mu \rightarrow e \gamma)<4.2 \times 10^{-13}
$$

As in the $\tau$-lepton case, the SM contribution to the $\mu \rightarrow e \gamma$ decay rate is negligible, due to the GIM suppression and tiny neutrino masses. Then, the upper bound in Eq. 107) can constrain the effective scale $\bar{m}_{L}^{21}$ defined above. In particular, one has

$$
\bar{m}_{L}^{21}>\left(\frac{96 \pi \alpha \mathrm{BR}_{\mu \rightarrow \nu_{\mu} \bar{\nu}_{e} e}^{\exp }}{\mathrm{BR}_{\mu \gamma}^{\max } G_{F}^{2} f_{1}\left(z_{e \mu}\right)}\right)^{1 / 4} \sqrt{\bar{F}_{L R}\left(x_{2}^{L}, \xi_{L}\right)},
$$

where $\mathrm{BR}_{\mu \gamma}^{\max } \equiv 4.2 \times 10^{-13}$, and one can assume $x_{2}^{L} \simeq\left(\frac{m_{e}}{m_{\mu}}\right)^{2} x_{3}^{L}$. Results are reported in Fig. 9. where we plotted $\bar{m}_{L}^{21}$ versus $x_{3}^{L}$. One can see that the constraints have a weak dependence on $x_{3}^{L}$, with $\bar{m}_{L}^{21} \lesssim(308-313) \mathrm{TeV}$ in the region $x_{3}^{L}<1-\xi_{L}$, for $\xi_{L} \gtrsim 0.1$. Indeed, since $x_{2}^{L} \simeq\left(\frac{m_{e}}{m_{\mu}}\right)^{2} x_{3}^{L} \ll 1$, the $x_{2}^{L}$ dependence of BR is almost flat in the range $x_{2}^{L} \ll 1$, due to the absence of $\log (x)$ infrared singularities for $x \rightarrow 0$ in $\bar{F}_{L R}(x, \xi)$.

By combining DM constraints on $\bar{m}_{L}$ with the ones from $\mu \rightarrow e \gamma$, considering the $\bar{m}_{L}$ lower 
bound from DM and vacuum stability for a few values of $\xi_{L}$, we get

$$
\begin{aligned}
\left|\frac{\tilde{\eta}_{L}^{12}}{\tilde{\eta}_{L}^{22}}\right|<3.4 \times 10^{-4}\left(\frac{\bar{m}_{L}}{5.7 \mathrm{TeV}}\right)^{2} \quad, \quad \text { for } \xi_{L}=0.1, \\
\left|\frac{\tilde{\eta}_{L}^{12}}{\tilde{\eta}_{L}^{22}}\right|<1.2 \times 10^{-5}\left(\frac{\bar{m}_{L}}{1.1 \mathrm{TeV}}\right)^{2}, \quad \text { for } \xi_{L}=0.5, \\
\left|\frac{\tilde{\eta}_{L}^{12}}{\tilde{\eta}_{L}^{22}}\right|<1.3 \times 10^{-6}\left(\frac{\bar{m}_{L}}{360 \mathrm{GeV}}\right)^{2} \quad, \quad \text { for } \xi_{L}=0.9 .
\end{aligned}
$$

Finally, from the $\mu \rightarrow e \gamma$ constraints, we obtain the following upper bounds

- for $\xi_{L}=0.1$ and $x_{3}^{L}=0.8$ (small-mixing regime)

$$
\mathrm{BR}^{(\mu \rightarrow e \gamma)}(\mu \rightarrow e \bar{\gamma})<3.9 \times 10^{-10}\left(\frac{\bar{\alpha}}{0.1}\right)
$$

- for $\xi_{L}=0.8$ and $x_{3}^{L}=0.1$ (large-mixing regime)

$$
\mathrm{BR}^{(\mu \rightarrow e \gamma)}(\mu \rightarrow e \bar{\gamma})<6.2 \times 10^{-10}\left(\frac{\bar{\alpha}}{0.1}\right)
$$

In Fig. 8 (right plot), we show the resulting $\mathrm{BR}(\mu \rightarrow e \bar{\gamma})$ expectations versus mixing, in the UF scenario. As before, the blue area corresponds to the allowed ranges, while the superimposed red area selects the regions excluded by the $\operatorname{BR}(\mu \rightarrow e \gamma)$ bounds. One can see that, for $\bar{e} \bar{e}_{3}^{L}=1, \tilde{\eta}_{L}^{12} / \tilde{\eta}_{L}^{22}=10^{-4}, \operatorname{BR}(\mu \rightarrow e \bar{\gamma})$ 's up to $\left(10^{-11}-10^{-10}\right)$ are allowed.

\section{Conclusions}

We have studied the FCNC decays of SM fermions into a dark photon, $f \rightarrow f^{\prime} \bar{\gamma}$, as foreseen by NP models with an extra unbroken $U(1)$ gauge group, acting on both a dark sector and a messenger sector, whose dynamics could explain the observed Yukawa coupling hierarchy. Model-dependent predictions for the corresponding BR's have been worked out, based on constraints given by DM abundance, vacuum stability, present non observation of non-SM states at the LHC, and bounds on the related radiative $f \rightarrow f^{\prime} \gamma$ decay rates.

We have found that large and possibly measurable BR's are allowed in most cases. In particular, for typical coupling strengths, predicted $\mathrm{BR}\left(f \rightarrow f^{\prime} \bar{\gamma}\right)$ 's reach $\sim\left(10^{-10}-10^{-7}\right)$ for the decays of top-quark, $\sim\left(10^{-4}-10^{-3}\right)$ for the $b$-quark, $\sim\left(10^{-8}-10^{-4}\right)$ for the $c$-quark, $\sim\left(10^{-10}-10^{-6}\right)$ for the $\tau$-lepton, and $\sim\left(10^{-11}-10^{-10}\right)$ for the $\mu$-lepton, depending on the mixing parameters and on the flavor-universality structure of the NP sector.

Most importantly, such decay channels are characterized by new peculiar two-body signatures, where a final SM fermion is balanced by a massless invisible ( $\nu$-like) system. The latter could be looked for at present and future colliders through dedicated searches, with high potential for either excluding large regions of the model parameter space or discovering a NP signal. 
For instance, large FCNC $t q \bar{\gamma}$ couplings might give rise to new signatures associated to top-quark production in high-energy collisions. Indeed, top-pair production at hadron colliders could be an ideal laboratory where to search for two-body $m_{t}$ resonances made up of a monochromatic jet and $\nu$-like missing energy/momentum associated to the undetected dark photon in the $t \rightarrow q \bar{\gamma}$ final state, where $E_{\text {jet }} \sim E_{\text {miss }} \sim m_{t} / 2$ in the top c.m. system.

An even more striking signature would correspond to the partonic $q g \rightarrow t \bar{\gamma}$ scattering, occurring via an $s$-channel $u$-, $c$-quark exchange. In this case a single top-quark system with unbalanced momentum would be associated to a massless invisible system. Such peculiar and clean collider top-quark signatures are not present in the SM, and possible backgrounds may arise only from particle and jet mismeasurements. Based on the BR upper bounds reported above, searches for FCNC top couplings to stable dark photons might indeed be explorable at future hadron colliders, like the FCC-hh, where a statistics of about $10^{12}$ top pairs could be available [19]. Note that the $\left(t+E_{\text {miss }}\right)$ final states are presently considered by LHC experiments in NP searches for massive invisible systems [40], [41].

As far as lighter flavors are concerned, the scenario looks even more promising. Huge and measurable values for $\operatorname{BR}(b \rightarrow q \bar{\gamma})$, where $q=s, d$, are presently allowed. Experimentally, as in the top-quark case, the $b \rightarrow q \bar{\gamma}$ is characterized by a peculiar signature, where the invisible massless dark photon equally shares the initial $b$-hadron energy and momentum with an $s$ - or $d$-initiated hadronic system. While hadron colliders are not the ideal place where to reconstruct such features, electron-positron $B$ factories [42] can offer the clean collision environment needed to control the invisible-system kinematical characteristics. An even better control could be available at future $Z$ factories (as possibly foreseen at the ILC [43], the FCC-ee [44, and the CEPC [45], running at the $Z$ peak), where the large boost of the $b$ hadrons could help in disentangling the invisible dark photon with high accuracy.

Similar features are shared by potentially measurable charm, tau, and muon decay rates into a dark photon, which can also be naturally scrutinized in $e^{+} e^{-}$collisions with large integrated luminosities. In particular, at the FCC-ee running on the $Z$ peak, clean samples of $\mathcal{O}\left(10^{11}-10^{12}\right)$ heavy-quark and lepton pairs of each given flavor from $Z \rightarrow f \bar{f}$ could be available [46], that, in absence of major systematics, could be sensitive to BR's into dark photons down to $\mathcal{O}\left(10^{-10}\right)$. Dedicated studies will be needed to accurately assess the actual sensitivity of present and future experiments to the FCNC and LFV fermion decay channels into a stable dark photon, naturally predicted in the theoretical NP framework considered in the present analysis.

\section{Acknowledgements}

EG would like to thank the CERN Theory Division for the kind hospitality during the preparation of this work. EV thanks the Theoretical Physics Department of the University of Trieste where part of this work has been done. This work was supported by the ERC Grant No. IUT23-6 and by the EU through the ERDF CoE program. 


\section{Appendix}

Here we present the analytical expressions for the NP contributions to the generic FCNC radiative decay amplitude corresponding to the process

$$
f^{i} \rightarrow f^{j} \gamma
$$

where $\gamma$ stands for a SM photon, and the indices $i, j(i>j$, with $i=3$ for the heaviest generation) both run on the fermion families either in the up or in the down $S U(2)_{L}$ sector. The Feynman diagrams contributing to this process are given by the diagrams (b) and (d) in Fig. 2, plus the usual flavor-changing self-energy (FCSE) contributions, that we do not show here. The FCSE graphs are required by gauge invariance, although not contributing to the $f^{i} \rightarrow f^{j} \gamma$ decay amplitude for an on shell photon, being the latter proportional to a flavorchanging magnetic-dipole operator. The $f^{i} \rightarrow f^{j} \gamma$ amplitude, for different $L / R$ chirality states, has the same structure as Eq. 28. for the $f^{i} \rightarrow f^{j} \bar{\gamma}$ amplitude, namely

$$
\begin{aligned}
M\left(f_{L}^{i} \rightarrow f_{R}^{j} \gamma\right) & =\frac{1}{\bar{\Lambda}_{L}^{f}}\left[\bar{u}_{R}^{j} \sigma_{\alpha \mu} u_{L}^{i}\right] q^{\mu} \epsilon^{\alpha}, \\
M\left(f_{R}^{i} \rightarrow f_{L}^{j} \bar{\gamma}\right) & =\frac{1}{\bar{\Lambda}_{R}^{f}}\left[\bar{u}_{L}^{j} \sigma_{\alpha \mu} u_{R}^{i}\right] q^{\mu} \epsilon^{\alpha},
\end{aligned}
$$

where $\epsilon^{\alpha}$ is the photon polarization vector. In the low energy approximation, the mass scales $\bar{\Lambda}_{L, R}^{f}$ do not depend on external momenta and can be worked out by matching the amplitude in Eq. (113) with the result of the full computation in the low energy limit. We neglect terms suppressed by loop factors and provide only the contributions proportional to the product $g_{L} g_{R}$. Then, similarly to Eqs. (29)-30) (with a different loop function), we obtain for the up quark sector

$$
\begin{aligned}
& \frac{1}{\left(\bar{\Lambda}_{L}^{U}\right)_{i j}}=\frac{m_{i}^{U}}{\bar{m}_{U}^{2}}\left(e e_{i}^{U} \frac{\rho_{R}^{j i}}{\rho_{R}^{i i}} \bar{F}_{L R}\left(x_{i}^{U}, \xi_{U}\right)\right), \\
& \frac{1}{\left(\bar{\Lambda}_{R}^{U}\right)_{i j}}=\frac{m_{i}^{U}}{\bar{m}_{U}^{2}}\left(e e_{i}^{U} \frac{\rho_{L}^{j i}}{\rho_{L}^{i i}} \bar{F}_{R L}\left(x_{i}^{U}, \xi_{U}\right)\right),
\end{aligned}
$$

and for the down quark sector

$$
\begin{aligned}
& \frac{1}{\left(\bar{\Lambda}_{L}^{D}\right)_{i j}}=\frac{m_{i}^{D}}{\bar{m}_{D}^{2}}\left(e e_{i}^{D} \frac{\eta_{R}^{j i}}{\eta_{R}^{i i}} \bar{F}_{L R}\left(x_{i}^{D}, \xi_{D}\right)\right), \\
& \frac{1}{\left(\bar{\Lambda}_{R}^{D}\right)_{i j}}=\frac{m_{i}^{D}}{\bar{m}_{D}^{2}}\left(e e_{i}^{D} \frac{\eta_{L}^{j i}}{\eta_{L}^{i i}} \bar{F}_{R L}\left(x_{i}^{D}, \xi_{D}\right)\right),
\end{aligned}
$$

where $e_{i}^{U(D)}$ are the EM charges of SM fermions in the up (down) sector, in unit of the EM charge $e$. The loop function $\bar{F}_{R L}(x, \xi)$ is given by

$$
\bar{F}_{L R}(x, \xi)=\bar{F}_{R L}(x, \xi)=\frac{\bar{f}_{2}(x, \xi)}{f_{1}(x, \xi)},
$$


where $f_{1}(x, \xi)$ is defined in Eq. 18$)$, and $\bar{f}_{2}(x, \xi)$ is given by

$$
\bar{f}_{2}(x, \xi)=\frac{1}{2 \xi}\left[\frac{(1+\xi)^{2}-x^{2}+2 x(1+\xi) \log \left(\frac{x}{1+\xi}\right)}{2(x-1-\xi)^{3}}-\{\xi \rightarrow-\xi\}\right] .
$$

In particular, the limits at small and large mixing are, respectively,

$$
\begin{aligned}
& \lim _{\xi \rightarrow 0} \bar{F}_{L R}(x, \xi)=\lim _{\xi \rightarrow 0} \bar{F}_{R L}(x, \xi)=\frac{1+4 x-5 x^{2}+2 x(2+x) \log x}{4(1-x)^{2}(1-x+x \log x)} \\
& \lim _{\xi \rightarrow 1} \bar{F}_{L R}(x, \xi)=\lim _{\xi \rightarrow 1} \bar{F}_{R L}(x, \xi)=\frac{(1-x)^{2}\left(4-8 x+3 x^{2}-2 x^{2} \log \frac{x}{2}\right)}{4 x(2-x)^{3}(1-x+x \log x)} .
\end{aligned}
$$

Contrary to the dark-photon loop function $F_{L R}(x, \xi)$ in Eq. $(32)$, the $\bar{F}_{L R}(x, \xi)$ expansion at $\xi \sim 1$ in Eq. (121) does not present $\log (1-\xi)$ singularities at the denominator.

\section{References}

[1] G. Aad et al. (ATLAS Collaboration), Phys. Lett. B 716, 1 (2012); S. Chatrchyan et al. (CMS Collaboration), Phys. Lett. B 716, 30 (2012).

[2] F. Englert and R. Brout, Phys. Rev. Lett. 13, 321 (1964); P. W. Higgs, Phys. Lett. 12, 132 (1964); P. W. Higgs, Phys. Rev. Lett. 13, 508 (1964); G. S. Guralnik, C. R. Hagen and T. W. B. Kibble, Phys. Rev. Lett. 13, 585 (1964).

[3] G. Aad et al. (ATLAS and CMS Collaborations), "Measurements of the Higgs boson production and decay rates and constraints on its couplings from a combined ATLAS and CMS analysis of the LHC $p p$ collision data at $\sqrt{s}=7$ and $8 \mathrm{TeV}$," J. High Energy Phys. 08 (2016) 045.

[4] E. Gabrielli and M. Raidal, Phys. Rev. D 89, 015008 (2014).

[5] E. Ma, Phys. Rev. Lett. 112, 091801 (2014); S. Fraser and E. Ma, Europhys. Lett. 108, $1002(2014)$.

[6] Y. Nambu and G. Jona-Lasinio, Phys. Rev. 122, 345 (1961); Y. Nambu and G. JonaLasinio, Phys. Rev. 124, 246 (1961).

[7] T. D. Lee and G. C. Wick, Phys. Rev. D 3, 1046 (1971). T. D. Lee and G. C. Wick, Phys. Rev. D 2, 1033 (1970).

[8] B. Grinstein, D. O'Connell, and M. B. Wise, Phys. Rev. D 77, 025012 (2008).

[9] B. Holdom, Phys. Lett. 166B, 196 (1986).

[10] E. Gabrielli, M. Heikinheimo, B. Mele, and M. Raidal, Phys. Rev. D 90, 055032 (2014). 
[11] S. Biswas, E. Gabrielli, M. Heikinheimo, and B. Mele, Phys. Rev. D 93, no. 9, 093011 (2016).

[12] S. Biswas, E. Gabrielli, M. Heikinheimo, and B. Mele, J. High Energy Phys. 06 (2015) 102.

[13] E. Gabrielli, Phys. Rev. D 77, 055020 (2008).

[14] K. A. Olive et al. (Particle Data Group Collaboration), Chin. Phys. C 38, 090001 (2014) and 2015 update.

[15] HFAG semileptonic group for the PDG 2014 Update, http://slac.stanford.edu/xorg/hfag/semi/pdg14/home.shtml

[16] S. L. Glashow, J. Iliopoulos, and L. Maiani, Phys. Rev. D 2, 1285 (1970).

[17] J. A. Aguilar-Saavedra and B. M. Nobre, Phys. Lett. B 553, 251 (2003).

[18] V. Khachatryan et al. (CMS Collaboration), J. High Energy Phys. HEP 04 (2016) 035.

[19] N. Arkani-Hamed, T. Han, M. Mangano, and L. T. Wang, "Physics Opportunities of a 100 TeV proton-proton Collider," Phys. Rep. 652, 1 (2016).

[20] M. Ciuchini, E. Gabrielli, and G. F. Giudice, Phys. Lett. B 388, 353 (1996); Phys. Lett. B 393, 489(E) (1997).

[21] J. F. Kamenik and C. Smith, J. High Energy Phys. 03 (2012) 090.

[22] A. J. Buras, "Climbing NLO and NNLO" summits of weak decays, arXiv:1102.5650, and references therein.

[23] M. Misiak et al., Phys. Rev. Lett. 114, 221801 (2015).

[24] G. Buchalla, A. J. Buras, and M. E. Lautenbacher, Rev. Mod. Phys. 68, 1125 (1996).

[25] K. G. Chetyrkin, M. Misiak, and M. Munz, Phys. Lett. B 400, 206 (1997); K. G. Chetyrkin, M. Misiak, and M. Munz, Phys. Lett. B 425, 414 (1998); A. J. Buras, A. Kwiatkowski, and N. Pott, Phys. Lett. B 414, 157 (1997); A. J. Buras, A. Kwiatkowski, and N. Pott, Phys. Lett. B 434, 459 (1998); C. Greub, T. Hurth, and D. Wyler, Phys. Rev. D 54, 3350 (1996); C. Greub and T. Hurth, Phys. Rev. D 56, 2934 (1997).

[26] A. F. Falk, M. E. Luke, and M. J. Savage, Phys. Rev. D 49, 3367 (1994);

[27] M. B. Voloshin, Phys. Lett. B 397, 275 (1997); A. Khodjamirian, R. Ruckl, G. Stoll, and D. Wyler, Phys. Lett. B 402, 167 (1997); Z. Ligeti, L. Randall, and M. B. Wise, Phys. Lett. B 402, 178 (1997); A. K. Grant, A. G. Morgan, S. Nussinov, and R. D. Peccei, Phys. Rev. D 56, 3151 (1997).

[28] E. Gabrielli, S. Khalil, and E. Torrente-Lujan, Nucl. Phys. B594, 3 (2001). 
[29] S. Chen et al. (CLEO Collaboration), Phys. Rev. Lett. 87, 251807 (2001).

[30] K. Abe et al. (Belle Collaboration), Phys. Lett. B 511, 151 (2001); A. Limosani et al. (Belle Collaboration), Phys. Rev. Lett. 103, 241801 (2009).

[31] B. Aubert et al. (BaBar Collaboration), Phys. Rev. D 77, 051103 (2008); J. P. Lees et al. (BaBar Collaboration), Phys. Rev. Lett. 109, 191801 (2012); J. P. Lees et al. (BaBar Collaboration), Phys. Rev. D 86, 112008 (2012); J. P. Lees et al. (BaBar Collaboration), Phys. Rev. D 86, 052012 (2012).

[32] Y. Amhis et al. [Heavy Flavor Averaging Group (HFAG) Collaboration], Averages of $b$ hadron, $c$-hadron, and $\tau$-lepton properties as of summer 2014, arXiv:1412.7515.

[33] A. Lenz et al., Phys. Rev. D 83, 036004 (2001).

[34] D. Becirevic et al., Nucl. Phys. B634, 105 (2002).

[35] A. Lenz, U. Nierste, J. Charles, S. Descotes-Genon, H. Lacker, S. Monteil, V. Niess, and S. T'Jampens, Phys. Rev. D 86, 033008 (2012).

[36] Z. Maki, M. Nakagawa, and S. Sakata, Prog. Theor. Phys. 28, 870 (1962); B. Pontecorvo, Zh. Eksp. Teor. Fiz. 34, 247 (1957). [Sov. Phys. JETP 7, 172 (1958)]

[37] J. Heeck, Phys. Lett. B 758, 101 (2016).

[38] B. Aubert et al. (BABAR Collaboration), Phys. Rev. Lett. 104, 021802 (2010).

[39] J. Adam et al. (MEG Collaboration), Phys. Rev. Lett. 110, 201801 (2013); A. M. Baldini et al. (MEG Collaboration), Eur. Phys. J. C 76, 434 (2016).

[40] G. Aad et al. (ATLAS Collaboration), Eur. Phys. J. C 75, 79 (2015).

[41] CMS Collaboration (CMS Collaboration), Search for new physics in a boosted hadronic monotop final state using $12.9 \mathrm{fb}^{-1}$ of $\sqrt{s}=13 \mathrm{TeV}$ data, Report No. CMS-PAS-EXO-16040 .

[42] B. Wang (Belle-II Collaboration), The Belle II Experiment and SuperKEKB upgrade, arXiv:1511.09434.

[43] H. Baer et al., The international linear collider technical design report - Volume 2: Physics, arXiv:1306.6352 [hep-ph].

[44] M. Bicer et al. [TLEP Design Study Working Group Collaboration], J. High Energy Phys. 01 (2014) 164.

[45] CEPC-SPPC Study Group, CEPC-SPPC Preliminary Conceptual Design Report. 1. Physics and Detector, Report No. IHEP-CEPC-DR-2015-01, IHEP-TH-2015-01, HEPEP-2015-01.

[46] P. Azzi, in FCC Week 2016, Rome 11-15 April 2016, http://fccw2016.web.cern.ch/fccw2016/. 